RASĀYAN J. Chem.

Vol. 13 | No. 3 |1853-1870| July - September | 2020 ISSN: 0974-1496 | e-ISSN: 0976-0083 | CODEN: RJCABP

\title{
STABLE ISONIAZID DERIVATIVES: IN SILICO STUDIES, SYNTHESIS AND BIOLOGICAL ASSESSMENT AGAINST Mycobacterium tuberculosis IN LIQUID CULTURE
}

\author{
S. L. Gaonkar ${ }^{1}$, S. S. Hakkimane ${ }^{2}$, B. R. Bharath ${ }^{3}$, V. P. Shenoy ${ }^{4}$, \\ U. N. Vignesh ${ }^{1}$ and B. R. Guru ${ }^{2, *}$ \\ ${ }^{1}$ Department of Chemistry, Manipal Institute of Technology, Manipal Academy of Higher \\ Education, Manipal-576104, (Karnataka) India \\ ${ }^{2}$ Department of Biotechnology, Manipal Institute of Technology, Manipal Academy of Higher \\ Education, Manipal-576104, (Karnataka) India \\ ${ }^{3}$ Atrimed Biotech LLP, Bangalore Bioinnovation Center, Biohelix park, Electronic City Phase 1, \\ Bangalore 560100, (Karnataka) India \\ ${ }^{4}$ Department of Microbiology, Kasturba Medical College, Manipal Academy of Higher \\ Education, Manipal-576104, (Karnataka) India \\ *E-mail: bharath.guru@manipal.edu
}

ABSTRACT

Isoniazid (INH) is well-known as a crucial drug in all multiple drug treatment for tuberculosis (TB) as approved by the WHO. It is a small molecule and highly hydrophilic, has low cellular penetration. By modifying highly hydrophilic drugs into hydrophobic will help in enhancing the cellular penetration of the drug and this, in turn, improves the therapeutic efficacy of the drug. Isoniazid a prodrug gets activated by KatG enzyme of Mycobacterium tuberculosis thus inhibiting InhA enzyme essential for the synthesis of Mycolic acid, a chief component of Mycobacterium tuberculosis cell wall. Hence in the current study, a series of isoniazid hydrazones are designed where Isoniazid can be modified to make it more stable in basic $\mathrm{pH}$ condition and InhA protein is identified as a potential drug target for Isoniazid derivatives to study the anti-tuberculosis activity. Active hydrazones were synthesized and characterized by modern analytical techniques and evaluated for their anti-tubercular activities. Antimicrobial susceptibility test (AST) against Mycobacterium tuberculosis was assessed using a different method Mycobacteria Growth Indicator Tube (MGIT ${ }^{\mathrm{TM}}$ ) 960 on a BACTEC system using molar equivalent concentrations of synthesized derivatives as recommended for minimal inhibitory concentration (MIC) of Isoniazid. The compounds in which INH modified with hydrophobic groups exhibited anti-tubercular activity on the H37Rv strain of Mycobacterium tuberculosis. The stability studies revealed that the synthesized INH derivatives were more stable than standard drug Isoniazid in basic $\mathrm{pH}$ conditions. MTT assay confirmed that synthesized active compounds do not pose any cytotoxic effect on RAW 264.7 macrophage cell line.

Keywords: Isoniazid, Isoniazid Hydrazones, Anti-tubercular Activity, Insilico Studies, Mycobacterium tuberculosis.

(C) RASĀYAN. All rights reserved

\section{INTRODUCTION}

Tuberculosis, a dominant respiratory disease of bacterial origin with high morbidity and mortality. Although it can be prevented and treated, it is the main cause of death globally. However, INH an isonicotinic acid-derived hydrazide has proved to be the most powerful antibiotic in the prevention and treatment of TB recommended by WHO. It has been used as a front-line antimycobacterial drug given for 6 months in both the phases of the treatment regime. Since M. tuberculosis is an intracellular organism, penetration of sufficient drug into the cell is required to clear the bacilli. INH is highly hydrophilic and is less stable in physiological $\mathrm{pH}$. Biological barriers are highly lipophilic because of which hydrophilic drugs have low membrane permeability requiring the administration of higher doses of drugs. Hematological alterations, vasculitis ${ }^{1}$ Peripheral neuropathy and Clinical hepatitis are the major adverse side effects of the

Rasayan J. Chem., 13(3), 1853-1870(2020)

http://dx.doi.org/10.31788/ RJC.2020.1335667

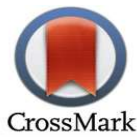


RASĀYAN J. Chem.

Vol. 13 | No. 3 |1853-1870| July - September | 2020

INH higher dose administration. ${ }^{2}$ Severe side effects due to the long treatment regimen result in low patient compliance for medication, rise to the emergence of MDR-TB. ${ }^{3}$ Hence, there is a need for modifying INH with hydrophobic moieties to enhance hydrophobicity and increase stability. This will increase the bioavailability to clear the intracellular M. tuberculosis bacilli. To overcome the problem, a class of derivatives of INH that exhibit efficient activity against bacilli is being developed. Researchers aim at exploring compounds that are potent anti-TB agents having minimal toxicity and maximum stability in comparison with those in use. ${ }^{4-7}$ The Antimycobacterial effect of INH was not known at the time of its discovery in the 1950s ${ }^{8,9}$ Studies in the 1970 s by Winder and Collins revealed the mechanism action of INH. INH enters the cell-wall of M. tuberculosis via passive diffusion and that its activity only against dividing bacteria. ${ }^{10-12}$ Similarly, the mechanism action of INH against M. tuberculosis has been studied from various perspectives. ${ }^{13}$ INH-nicotinamide adenine dinucleotide (NAD) adduct are formed by activation of KatG (Multifunctional enzyme) arresting InhA and enoyl-acyl carrier protein (ACP) reductase, enzymes necessary for the production of mycolic acids which is a major component of cell wall. ${ }^{14}$ ${ }^{15}$ The in silico study suggests that redesigning INH to modified INH is a good approach to overcome resistance in KatG that can accomplish the pharmacological effect of INH. ${ }^{16}$ Likewise, extensive pharmacological functionality, structural flexibility and ligating behavior of hydrazones have gained extensive attention. Hydrazones are notable for their two interlinked nitrogen atoms CN-NR2, which possess antimicrobial, antitumor and antitubercular properties. ${ }^{17,18}$ Reports on derivatives of Isonicotinoyl hydrazone containing heterocyclic moiety exhibiting anti-tubercular activity, ${ }^{19,20}$ similarly various studies on the antimicrobial activity of alkyl chains, anti-fungal, and toxicity of various INH derivatives were also reported. ${ }^{21-23}$ Improving the efficiency of existing drugs by synthetic chemical alterations, to obtain a new series of compounds with better action against microbial resistance has become the need of the hour. Modification of existing effective drugs such as INH that are probable intermediates responsible for its antimycobacterial activity and testing them using anti-mycobacterial drug susceptibility tests will become more beneficial. In our lab, INH was reformed into Isoniazid benzhydrazine (IH2) and loaded into PLGA nanoparticles. The modified compound was stable and increased the drug loading by 15 -folds greater than INH in PLGA nanoparticles. ${ }^{24}$ Prompted by this result we have developed a series of isoniazid hydrazones, in continuation of the work on the development of bioactive heterocycles. ${ }^{25-29}$ Our experimental results emphasize the preparation, characterization and docking studies to know the activity of INH hydrazine derivatives theoretically. Evaluation of INH derivatives for anti-tubercular activity using BACTEC MGIT ${ }^{\text {TM }} 960$ liquid culture against H37Rv strain. Presently, MGITTM 960 system is referred to as the gold standard in a liquid culture, known for detection, drug susceptibility, and analysis of M. tuberculosis. ${ }^{30,31}$ The growth positives were signaled by indications based on particular growth algorithms. Biologically active INH derivatives were analyzed for cytotoxic effect on macrophage RAW 264.7 cell line. The stability of the synthesized compounds was assessed under specific physiological conditions and compared it with standard INH.

\section{EXPERIMENTAL}

\section{In-silico Studies}

\section{Target Identification}

KatG activates INH resulting in the formation of isonicotinic acyl radical, which further couples to NADH forming adduct and attaches firmly to the InhA to obstruct enoyl-AcpM substrate and synthesize fatty acids, hence preventing synthesis of mycolic acids (Vital constituents of the cell wall). With the activation of KatG of INH few radicals along with nitric oxide are obtained signifying the action of another antimycobacterial prodrug Pretomanid. ${ }^{32,33}$ Hence InhA protein is identified as a potential drug target for INH derivatives to bring in the anti-tuberculosis activity.

\section{Molecular Docking}

The crystal structure of InhA protein, the known target for INH to combat against M. tuberculosis (PDB ID: 1ENY, $2.2 \AA$ resolution) was achieved from the protein data bank (Fig.1) and set by the Protein Preparation Wizard ${ }^{34}$ accessible in Schrödingèr suite 2018-1. Removal and addition of Crystallographic water molecules (without $3 \mathrm{H}$ bonds) were removed and hydrogen bonds at $\mathrm{pH} 7.0$ were added considering the appropriate ionization conditions for the basic and acidic amino acid residues. Addition of missing side- 
RASĀYAN J. Chem.

Vol. 13 | No. 3 |1853-1870| July - September | 2020

chain atoms and breaks exist in the structure were constructed with Prime v3.7, Schrödingèr 2018-1. ${ }^{35}$ The crystal structure energy was reduced by the OPLS--2005 force field..$^{36}$ The active site defines the presence of a $10 \AA$ radius around the ligand (INH) in the crystal structure and created a grid box for docking towards the centroid of the active site. The Grid-Based Ligänd Docking with Energetics Glidè v7.8, Schrödingër 2018-1 was employed to predict low energy conformations of compounds docked in the catalytic pocket. ${ }^{37}$ The final best-docked molecules were marked using a Glide score function, Glide energy and Glide E model energy. Finally, the lowest-energy docked complex of 12/1ENY was subjected to Molecular MechanicsGeneralized Born Surface Area (MM-GBSA). Local optimization feature in Prime v3.7, Schrödingèr 2018$1^{35}$ was used to decrease the docked poses and binding free energies for complexes were calculated by Molecular MM-GBSA using OPLS_2005 force field, ${ }^{38}$ VSGB solvent model ${ }^{38}$ and rotamer search algorithms.

\section{Molecular Dynamics (MD) Stimulation}

The $\mathrm{MD}^{39}$ of the docked complex of 12 with $1 \mathrm{ENY}$ was determined by the OPLS- 2005 force field $^{36}$ in an obvious solvent through the TIP3P model ${ }^{40}$ of water using the Desmond software. The 1ENY/366353 complex was solvated in an orthorhombic box ( $\AA 3)$ permitting a $10 \AA$ of buffer region. The built system was consisting of 35,315 (which includes 31,266 water molecules) atoms and the protein-substrate complex was constituted by 4009 atoms. The LBFGS minimization was done to attain a gradient threshold of 25 $\mathrm{kcal} / \mathrm{mol} / \AA$ by a minimum of 3 vectors and 10 steepest descent steps. With the convergence threshold retained at $1.0 \mathrm{kcal} / \mathrm{mol} / \AA$, minimization was done for a maximum of 2000 iterations $\mathrm{s}^{41}$. The molecular system remained heated in the NPT ensemble to $300^{\circ} \mathrm{K}$ keeping a time step of $2 \mathrm{fs}$ and the MD was run for $10 \mathrm{~ns}$ using a Nose-Hoover thermostat. ${ }^{42,43} \mathrm{MD}$ data were captured for every $100 \mathrm{ps}$ and trajectories were checked visually.

\section{Chemistry}

The reagents used in the synthesis, as well as analysis, were of synthetic grade and analytical grade obtained commercially. Uncorrected melting point values of the compounds were noted. The IR spectroscopy was performed on a Shimadzu-8300 FTIR Spectrometer using $\mathrm{KBr}$ pellets values represented in $v_{\max }, \mathrm{cm}^{-1}$. The ${ }^{1} \mathrm{H}-\mathrm{NMR}$ and ${ }^{13} \mathrm{C}-\mathrm{NMR}$ spectrum of synthesized compounds were determined on a Bruker $400 \mathrm{MHz}, 100$ MHz NMR Spectrophotometer respectively in $d_{6}-D M S O$. The chemical shifts are stated in the $\delta$ ppm scale. The Agilent 6520 Accurate-Mass Q TOF LC/MS was used to record Mass Spectra. The purity of the samples was determined by HPLC (Shimadzu LC-20AD with UV detector and autosampler SPD-20A was initialized by Column C 18 Luna, $150 \mathrm{~mm}, 5 \mathrm{u}, 100 \AA$ and mobile phase Methanol: Water. Gradient elution with $15 \mathrm{~min}$ runtime, detected at $268 \mathrm{~nm} / 290 \mathrm{~nm}$ and injection volume used was $20 \mu 1$. Thin Layer Chromatography (TLC) was performed on silica gel (HF254 200 mesh) using chloroform: ethyl acetate and visualized in the UV chamber.

\section{Synthesis of Ethyl Isonicotinate, 2}

A parallel synthesizer equipped with a magnetic stirrer was introduced with $1 \mathrm{~g}$ isonicotinic acid $1,3 \mathrm{~mL}$ of ethanol and 2 drops of Conc. $\mathrm{H}_{2} \mathrm{SO}_{4}$. The reaction mass was maintained under reflux for 2 hours. Completion of reaction was monitored (TLC method, Chloroform: ethyl acetate $=7.5: 2.5$ ). The excess ethanol was distilled, and the mass left was dissolved in ether $(10 \mathrm{~mL})$, washed with $5 \% \mathrm{NaHCO}_{3}$, water and dried. Ether was evaporated to get the ethyl isonicotinate $1.2 \mathrm{~g}(98 \%)$ which was taken for the next step.

\section{Synthesis of Isoniazid, 3}

A parallel synthesizer equipped with a magnetic stirrer was charged with ethyl isonicotinate $2,(1.2 \mathrm{~g}, 10$ mmol), hydrazine hydrate and ethanol ( 1 and $2 \mathrm{~mL}$ respectively). The reaction was heated at $70^{\circ} \mathrm{C}$ for two hours. Reaction completion was examined by TLC. The mass was cooled and stirred at room temperature and crystals formed were filtered to give isoniazid 3 to yield $1.03 \mathrm{~g}(92 \%)$. 
Synthesis of N-[(Substituted aromatic) methylidene] pyridine-4-carbohydrazides, 5 (a-m)

A parallel synthesizer equipped with a magnetic stirrer was charged with an equimolar mixture of isoniazid 3, aromatic aldehyde $4(\mathbf{a}-\mathbf{m})$ and isopropyl alcohol (5vol). The reaction mass was stirred at $70{ }^{\circ} \mathrm{C}$ for $1 \mathrm{~h}$. TLC (Chloroform: ethyl acetate $=7.5: 2.5$ ) was used to monitor the progress of the reaction. The obtained product was cooled and the solid was filtered, recrystallized by ethanol after the completion of the reaction. ${ }^{44-48}$

\section{N'-[phenylmethylidene] pyridine-4-carbohydrazide (5a)}

It was obtained from Isoniazid $0.14 \mathrm{~g}(1 \mathrm{mmol})$, benzaldehyde $0.1 \mathrm{~g}(1 \mathrm{mmol})$ as a white solid to yield 0.15 $\mathrm{g}(69 \%)$, m.p $=200-202{ }^{\circ} \mathrm{C}$, HPLC purity: 99. \%. Infrared : $1558(\mathrm{C}=\mathrm{N}), 1681(\mathrm{CO}), 3028$ (Aromatic CH), 3395(-NH) (Fig. S1). ${ }^{1} \mathrm{H}$ NMR : 7.4-7.5 (m, 3H, Aromatic H), 7.7 (d, 2H, Aromatic H), 7.8 (d, 2H, Aromatic $\mathrm{H}), 8.55(\mathrm{~s}, 1 \mathrm{H},-\mathrm{N}=\mathrm{CH}), 8.8(\mathrm{~d}, 2 \mathrm{H}, \mathrm{ArH}), 12.1(\mathrm{~s}, 1 \mathrm{H},-\mathrm{NH})\left(\right.$ Fig. S2). ${ }^{13} \mathrm{C}$ NMR : 122.0, 127.3, 129.3,

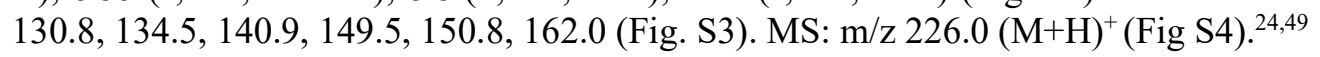

\section{$\mathbf{N}^{\prime}$-[(3-nitrophenyl)methylidene]pyridine-4-carbohydrazide (5b)}

It was obtained from Isoniazid $0.14 \mathrm{~g}(1 \mathrm{mmol}), 3$-nitrobenzaldehyde $0.15 \mathrm{~g}(1 \mathrm{mmol})$ as a white solid to yield $0.19(70 \%), \mathrm{m} . \mathrm{p}=234-236^{\circ} \mathrm{C}$, HPLC purity: $99.2 \%$. IR : $3337(\mathrm{NH}), 3036(\mathrm{ArCH}), 1667(\mathrm{CO})$, $1531(\mathrm{C}=\mathrm{N}), 1596\left(\mathrm{NO}_{2}\right) .{ }^{1} \mathrm{H}$ NMR : $\delta$ ppm: $7.3(\mathrm{~d}, 1 \mathrm{H}$, Aromatic $\mathrm{H}), 7.4(\mathrm{t}, 1 \mathrm{H}$, Aromatic $\mathrm{H}), 7.5(\mathrm{~d}, 1 \mathrm{H}$, Aromatic $\mathrm{H}), 7.7(\mathrm{~s}, 1 \mathrm{H}$, Aromatic $\mathrm{H}), 7.8(\mathrm{~d}, 2 \mathrm{H}$, Aromatic $\mathrm{H}), 8.4(\mathrm{~s}, 1 \mathrm{H}, \mathrm{CH}), 8.8(\mathrm{~d}, 2 \mathrm{H}$, Aromatic $\mathrm{H})$, $12.1(\mathrm{~s}, 1 \mathrm{H},-\mathrm{NH}) .{ }^{13} \mathrm{C}$ NMR : $124.1,126.2,127.9,128.8,129.9,130.1,133.1,144.3,149.0,150.2,162.3$. MS: $\mathrm{m} / \mathrm{z} 271(\mathrm{M}+\mathrm{H})^{+}$.

\section{$\mathbf{N}^{\prime}-\left[\left(\left[1,1^{\prime}\right.\right.\right.$-biphenyl]-4-yl)methylidene]pyridine-4-carbohydrazide (5c)}

It was obtained from isoniazid $0.14 \mathrm{~g}(1 \mathrm{mmol})$, biphenyl-4-carboxaldehyde $0.18 \mathrm{~g}(1 \mathrm{mmol})$, as a white solid to yield $0.204 \mathrm{~g}(68 \%), \mathrm{m} . \mathrm{p}=216-218^{\circ} \mathrm{C}$, HPLC purity: $98.6 \%$ ( Fig. S5). Infrared : $1541(\mathrm{C}=\mathrm{N}$ ), $1653(\mathrm{C}=\mathrm{O}), 3012$ (Aromatic CH), 3464 (NH) (Fig. S6). ${ }^{1} \mathrm{H}$ NMR : 7.3-7.5 (m, 3H, AromaticH), 7.7 (d, $2 \mathrm{H}$, Aromatic H), $7.8(\mathrm{~d}, 2 \mathrm{H}$, Aromatic H), 7.81-7.86 (m, 4H, Aromatic H), $8.5(\mathrm{~s}, 1 \mathrm{H}, \mathrm{CH}), 8.8(\mathrm{~d}, 2 \mathrm{H}$, Aromatic H), 12.11 (s, 1H, NH) (Fig. S7). ${ }^{13} \mathrm{C}$ NMR 122.0, 127.1, 127.5, 128.3, 128.4, 129.5, 133.6, 139.7, 140.9, 142.3, 149.0, 150.8, 162.0 (Fig. S8). MS: m/z $302(\mathrm{M}+\mathrm{H})^{+}$.

\section{N'-[(4-methylphenyl)methylidene]pyridine-4-carbohydrazide (5d)}

It was obtained from isoniazid $0.14 \mathrm{~g}(1 \mathrm{mmol}), 4$-methylbenzaldehyde $0.12 \mathrm{~g}(1 \mathrm{mmol})$ as a white crystal to yield $0.17 \mathrm{~g}(71 \%), \mathrm{m} . \mathrm{p}=248-250{ }^{\circ} \mathrm{C}$ HPLC purity: $98.5 \%$ (Fig S9). Infrared : $1558(\mathrm{C}=\mathrm{N}), 1662$ ( $\mathrm{C}=\mathrm{O}), 3015$ (Aromatic $\mathrm{CH}), 3371(\mathrm{NH})(\mathrm{Fig} \mathrm{S10}) .{ }^{1} \mathrm{H} \mathrm{NMR}: 2.82\left(\mathrm{~s}, 3 \mathrm{H}, \mathrm{CH}_{3}\right), 6.87(\mathrm{~d}, 2 \mathrm{H}$, AromaticH), 7.75(d, 2H, Aromatic H), $7.86(\mathrm{~d}, 2 \mathrm{H}$, Aromatic H), $8.53(\mathrm{~s}, 1 \mathrm{H}, \mathrm{CH}), 8.71(\mathrm{~d}, 2 \mathrm{H}$, Aromatic H), 12.21 (s, $1 \mathrm{H}, \mathrm{NH}) .{ }^{13} \mathrm{C}$ NMR : $21.3,127.3,128.3129 .5,130.1,130.7,133.2,149.3,150.4,162.4 . \mathrm{MS}: \mathrm{m} / \mathrm{z} 240.11$ $(\mathrm{M}+\mathrm{H})^{+}$.

\section{N'-[(4-chlorophenyl)methylidene]pyridine-4-carbohydrazide (5e)}

It was obtained from isoniazid $0.14 \mathrm{~g}(1 \mathrm{mmol})$, 4-chlorobenzaldehyde $0.14 \mathrm{~g}(1 \mathrm{mmol})$ as a white crystal to yield $0.17 \mathrm{~g}(65 \%)$, m.p. $=206-208^{\circ} \mathrm{C}$. HPLC purity: 99\%. Infrared : $690(\mathrm{CCl}), 1545(\mathrm{C}=\mathrm{N}), 1660(\mathrm{C}=\mathrm{O})$, 3044 (Aromatic CH), $3460(\mathrm{NH}) .{ }^{1} \mathrm{H}$ NMR : 7.39 (d, 2H, Aromatic H), $7.52(\mathrm{~d}, 2 \mathrm{H}$, Aromatic H), $7.72(\mathrm{~d}$, $2 \mathrm{H}$, Aromatic H), $8.43(\mathrm{~s}, 1 \mathrm{H}, \mathrm{CH}), 8.75\left(\mathrm{~d}, 2 \mathrm{H}\right.$, Aromatic H), $12.21(\mathrm{~s}, 1 \mathrm{H}, \mathrm{NH}) .{ }^{13} \mathrm{C} \mathrm{NMR}: 127.1,128.9$, $129.1,129.8,130.0,135.4,149.3,150.5,162.2 . \mathrm{MS}: \mathrm{m} / \mathrm{z} 260.5(\mathrm{M}+\mathrm{H})^{+}$.

\section{N'-[(4-fluorophenyl)methylidene]pyridine-4-carbohydrazide (5f)}

It was obtained from isoniazid $0.14 \mathrm{~g}(1 \mathrm{mmol})$, 4-Fluorobenzaldehyde $0.12 \mathrm{~g}(1 \mathrm{mmol})$ as a white crystal to yield $0.17 \mathrm{~g} \mathrm{(71 \% ),} \mathrm{m.p.} 170-172{ }^{\circ} \mathrm{C}$. HPLC purity: $98 \%$. Infrared : $1134(\mathrm{CF}), 1512(\mathrm{C}=\mathrm{N}), 1670(\mathrm{C}=\mathrm{O})$, 3025 (Aromatic CH), $3391(\mathrm{NH}) .{ }^{1} \mathrm{H}$ NMR : $7.61(\mathrm{~d}, 2 \mathrm{H}$, Aromatic H), $7.72(\mathrm{~d}, 2 \mathrm{H}$, Aromatic H), $7.85(\mathrm{~d}$, $2 \mathrm{H}$, Aromatic H), $8.41(\mathrm{~s}, 1 \mathrm{H} \mathrm{CH}), 8.72(\mathrm{~d}, 2 \mathrm{H}$, Aromatic $\mathrm{H}), 12.12(\mathrm{~s}, 1 \mathrm{H}, \mathrm{NH}) .{ }^{13} \mathrm{C} \mathrm{NMR}: 115.8,128.2$, $129.9,130.5,132.6,149.4,150.2,162.1,163.4$. MS: $\mathrm{m} / \mathrm{z} 244(\mathrm{M}+\mathrm{H})^{+}$. 
RASĀYAN J. Chem.

Vol. 13 | No. 3 |1853-1870| July - September | 2020

\section{N'-[(2-hydroxyphenyl)methylidene]pyridine-4-carbohydrazide (5g)}

It was obtained from isoniazid $0.14 \mathrm{~g}(1 \mathrm{mmol})$, salicylaldehyde $0.12 \mathrm{~g}(1 \mathrm{mmol})$ as a white powder to yield $0.16 \mathrm{~g}(66 \%)$, m.p. $250-252^{\circ} \mathrm{C}$. HPLC purity: $98 \%$. Infrared : $1562(\mathrm{C}=\mathrm{N}), 1678(\mathrm{C}=\mathrm{O}), 3009$ (Aromatic $\mathrm{CH}), 3186(\mathrm{NH}), 3456(\mathrm{OH}) .{ }^{1} \mathrm{H}$ NMR $6.94(\mathrm{~m}, 2 \mathrm{H}$, Aromatic H), $7.3(\mathrm{t}, 1 \mathrm{H}$, Aromatic H), $7.62(\mathrm{~d}, 1 \mathrm{H}$, Aromatic $\mathrm{H}), 7.85(\mathrm{~d}, 2 \mathrm{H}$, Aromatic $\mathrm{H}), 8.6(\mathrm{~s}, 1 \mathrm{H}, \mathrm{CH}), 8.80(\mathrm{~d}, 2 \mathrm{H}$, Pyridine $\mathrm{H}), 11.1(\mathrm{~s}, 1 \mathrm{H}, \mathrm{OH}), 12.3$ (s, $1 \mathrm{H}, \mathrm{NH}) .{ }^{13} \mathrm{C}$ NMR $116.6,119.1,119.9,121.9,129.7,132.2,140.4,149.5,150.8,157.9,161.8$. MS: $\mathrm{m} / \mathrm{z} 242(\mathrm{M}+\mathrm{H})^{+}$.

\section{$\mathbf{N}^{\prime}$-[(2,5-dimethoxyphenyl)methylidene]pyridine-4-carbohydrazide (5h)}

It was obtained from isoniazid $0.14 \mathrm{~g}(1 \mathrm{mmol}), 2,5$-Dimethoxybenzaldehyde $0.17 \mathrm{~g}(1 \mathrm{mmol})$ as yellow crystals to yield $0.205 \mathrm{~g}(74 \%), \mathrm{m} . \mathrm{p}=260-262{ }^{\circ} \mathrm{C}$. HPLC purity $99 \%$. Infrared : $1550(\mathrm{C}=\mathrm{N}), 1654($ $\mathrm{C}=\mathrm{O}), 3035($ Aromatic $\mathrm{CH}), 3351(\mathrm{NH}) .{ }^{1} \mathrm{H}$ NMR $3.7\left(\mathrm{~s}, 3 \mathrm{H},-\mathrm{OCH}_{3}\right), 3.8\left(\mathrm{~s}, 3 \mathrm{H},-\mathrm{OCH}_{3}\right), 7.02-7.09(\mathrm{~m}$, $2 \mathrm{H}$, AromaticH), $7.38(\mathrm{~d}, 1 \mathrm{H}$, Aromatic $\mathrm{H}), 7.83(\mathrm{~d}, 2 \mathrm{H}$, Aromatic $\mathrm{H}), 8.78(\mathrm{~d}, 2 \mathrm{H}$, Aromatic $\mathrm{H}), 8.8(\mathrm{~s}$, $1 \mathrm{H}, \mathrm{CH}=\mathrm{N}), 12.0(\mathrm{~s}, 1 \mathrm{H}, \mathrm{NH}) .{ }^{13} \mathrm{C} \mathrm{NMR}: 49.0,55.9,109.7,113.9,118.5,121.9,123.6,140.3,144.8,149.9$, 150.7, 152.9, 161.8. MS: m/z $286(\mathrm{M}+\mathrm{H})^{+}$.

\section{N'-[(3,4-dihydroxyphenyl)methylidene]pyridine-4-carbohydrazide (5i)}

It was obtained from isoniazid $0.14 \mathrm{~g}(1 \mathrm{mmol}), 3,4$-Dihydrobenzaldehyde $0.14 \mathrm{~g}(1 \mathrm{mmol})$ as a white powder to yield $0.18 \mathrm{~g}(71 \%), \mathrm{m} . \mathrm{p}=202-204^{\circ} \mathrm{C}$. HPLC $99 \%$. Infrared : $1546(\mathrm{C}=\mathrm{N}), 1651(\mathrm{C}=\mathrm{O}), 3071$ (Aromatic CH), $3474(\mathrm{OH}) .{ }^{1} \mathrm{H}$ NMR : $7.61(\mathrm{~s}, 1 \mathrm{H}$, Aromatic H), $7.68(\mathrm{~d}, 1 \mathrm{H}$, Aromatic H), $7.71(\mathrm{~d}, 1 \mathrm{H}$, Aromatic $\mathrm{H}), 7.83(\mathrm{~d}, 2 \mathrm{H}$, Aromatic $\mathrm{H}), 8.42(\mathrm{~s}, 1 \mathrm{H}, \mathrm{CH}), 8.81(\mathrm{~d}, 2 \mathrm{H}$, Aromatic $\mathrm{H}), 11.09(\mathrm{~s}, 1 \mathrm{H}, \mathrm{OH})$, $11.21(\mathrm{~s}, 1 \mathrm{H}, \mathrm{OH}), 12.12(\mathrm{~s}, 1 \mathrm{H}, \mathrm{NH}) .{ }^{13} \mathrm{C} \mathrm{NMR} 116.6,118.1,121.8,123.3,131.3,140.8,146.1,146.8$, 149.1, 149.9, 164.1. MS: m/z $258(\mathrm{M}+\mathrm{H})^{+}$.

\section{$\mathbf{N}^{\prime}$-[(1H-indol-3-yl)methylidene]pyridine-4-carbohydrazide (5j)}

It was obtained from isoniazid $0.14 \mathrm{~g}(1 \mathrm{mmol})$, Indole-3-carbaldehyde, $0.14 \mathrm{~g}(1 \mathrm{mmol})$ as a pale brown powder to yield $0.18 \mathrm{~g}(69 \%)$, m.p. $220-222^{\circ} \mathrm{C}$. HPLC $97.7 \%$. Infrared : $1546(\mathrm{C}=\mathrm{N}), 1658(\mathrm{C}=\mathrm{O}), 3028$ (-Aromatic CH), $3394(\mathrm{NH}), 3545$ (indole NH). ${ }^{1} \mathrm{H}$ NMR : 7.12-7.23 (m, 2H, Aromatic H), $7.44(\mathrm{~d}, 1 \mathrm{H}$, Aromatic $\mathrm{H}), 7.82(\mathrm{~d}, 2 \mathrm{H}$, Aromatic $\mathrm{H}), 7.88(\mathrm{~d}, 1 \mathrm{H}$, Aromatic $\mathrm{H}), 8.26(\mathrm{~d}, 1 \mathrm{H}$, Aromatic $\mathrm{H}), 8.62(\mathrm{~s}, 1 \mathrm{H}$, $\mathrm{CH}), 8.78\left(\mathrm{~d}, 2 \mathrm{H}\right.$, Aromatic H), $11.65(\mathrm{~s}, 1 \mathrm{H}, \mathrm{NH}), 11.75(\mathrm{~s}, 1 \mathrm{H}, \mathrm{NH}) .{ }^{13} \mathrm{C}$ NMR 110.9, 112.2, 119.9, 121.6, $121.9,122.1,127.3,130.9,137.3,140.2,143.3,149.9,163.5 . \mathrm{MS}: \mathrm{m} / \mathrm{z} 265(\mathrm{M}+\mathrm{H})^{+}$.

\section{N'-[(4-\{2-[methyl(pyridin-2-yl)amino]ethoxy\}phenyl)methylidene]pyridine-4-carbohydrazide (5k)}

It was obtained from isoniazid $0.14 \mathrm{~g}(1 \mathrm{mmol}), 4-\{2$-[methyl(pyridin-2-yl)amino] ethoxy $\}$ benzaldehyde $0.25 \mathrm{~g}(1 \mathrm{mmol})$ as a pale green crystal to yield $0.273 \mathrm{~g}(74 \%), \mathrm{m} . \mathrm{p}=128-130^{\circ} \mathrm{C}$. HPLC purity $98.6 \%$ (Fig. S1 1). Infrared : $1504(\mathrm{C}=\mathrm{N}), 1656(\mathrm{C}=\mathrm{O}), 3065(\mathrm{CH}), 3316(\mathrm{NH})$ (Fig. S12). ${ }^{1} \mathrm{H}$ NMR : 3.94 (s, 3H, $\mathrm{Me}), 4.19\left(\mathrm{t}, 2 \mathrm{H}, \mathrm{CH}_{2}\right), 4.22\left(\mathrm{t}, 2 \mathrm{H}, \mathrm{CH}_{2}\right), 6.55(\mathrm{t}, 1 \mathrm{H}$, Aromatic $\mathrm{H}), 6.69(\mathrm{~d}, 1 \mathrm{H}$, Aromatic $\mathrm{H}), 7.06(\mathrm{~d}, 2 \mathrm{H}$, Aromatic $\mathrm{H}), 7.48(\mathrm{~d}, 1 \mathrm{H}$, Aromatic $\mathrm{H}), 7.51(\mathrm{~d}, 2 \mathrm{H}$, Aromatic $\mathrm{H}), 7.64(\mathrm{~d}, 2 \mathrm{H}$, Aromatic $\mathrm{H}), 7.83(\mathrm{~d}, 1 \mathrm{H}$, Aromatic H), 8.09 (s, 1H, CH), 8.74 (d, 2H, Aromatic H), 11.9 (s, 1H, NH) (Fig. S13). ${ }^{13} \mathrm{C}$ NMR 35.6, 61.5, 67.6, 109.2, 114.8, 118.2, 121.9, 125.5, 129.9, 138.8, 140.8, 147.2, 148.3, 149.8, 154.3, 162.0, 164.4. MS: $\mathrm{m} / \mathrm{z} 376(\mathrm{M}+\mathrm{H})^{+}$.

\section{N'-[(2-butyl-5-chloro-1H-imidazol-4-yl)methylidene]pyridine-4-carbohydrazide (5l)}

It was obtained from isoniazid $0.14 \mathrm{~g}(1 \mathrm{mmol}), 2$-butyl-5-chloro-1H-imidazole-4-carbaldehyde $0.17 \mathrm{~g}(1$ mmol) as a pale yellow crystal to yield $0.204 \mathrm{~g}(68 \%), \mathrm{m} . \mathrm{p}=148-150{ }^{\circ} \mathrm{C}$. HPLC purity $99 \%$ (Fig. S14). Infrared : $686(\mathrm{CCl}), 1558(\mathrm{C}=\mathrm{N}), 1670(\mathrm{C}=\mathrm{O}) 3055$ (Aromatic $\mathrm{CH}), 3182$ (amide $\mathrm{NH}), 3362$ (imidazole $\mathrm{NH})\left(\right.$ Fig. S15). ${ }^{1} \mathrm{H}$ NMR : $1.33\left(\mathrm{t}, 3 \mathrm{H}, \mathrm{CH}_{3}\right), 1.5-1.60\left(\mathrm{~m}, 2 \mathrm{H}, \mathrm{CH}_{2}\right), 1.6-1.65\left(\mathrm{~m}, 2 \mathrm{H}, \mathrm{CH}_{2}\right), 2.50(\mathrm{t}, 2 \mathrm{H}$, $\left.\mathrm{CH}_{2}\right), 7.82(\mathrm{~d}, 2 \mathrm{H}$, Aromatic H), $8.34(\mathrm{~s}, 1 \mathrm{H}, \mathrm{CH}), 8.78(\mathrm{~d}, 2 \mathrm{H}$, Aromatic H), $11.94(\mathrm{~s}, 1 \mathrm{H}, \mathrm{NH}), 12.95(\mathrm{~s}$, 1H, NH) (Fig. S16). ${ }^{13}$ C NMR 13.2, 23.2, 28.3, 30.2, 116.4, 121.8, 128.2, 139.6, 140.7, 148.2, 149.8, 164.4. MS: $\mathrm{m} / \mathrm{z} 306(\mathrm{M}+\mathrm{H})^{+}$. 
RASĀYAN J. Chem.

Vol. 13 | No. 3 |1853-1870| July - September | 2020

\section{$\mathbf{N}^{\prime}$-[phenyl 1,2-dimethylidene]pyridine-4-carbohydrazide (5m)}

It was obtained from isoniazid $0.14 \mathrm{~g}(1 \mathrm{mmol})$, phenyl 1,2-dicarbaldehyde $0.27 \mathrm{~g}(2 \mathrm{mmol})$ as a white powder to yield $0.26 \mathrm{~g}(69 \%), \mathrm{m} . \mathrm{p}=158-160{ }^{\circ} \mathrm{C}$. HPLC purity $98 \%$ (Fig. S17). Infrared : $1556(\mathrm{C}=\mathrm{N}$ ), $1662(\mathrm{C}=\mathrm{O}), 3075($ Aromatic $\mathrm{CH}), 3262(\mathrm{NH}) .{ }^{1} \mathrm{H}$ NMR : $7.56(\mathrm{~d}, 1 \mathrm{H}$, Aromatic H), 7.81(d, 2H, Pyridine $\mathrm{H}), 7.90\left(\mathrm{~d}, 1 \mathrm{H}\right.$, Aromatic H), $8.80\left(\mathrm{~d}, 2 \mathrm{H}\right.$, Pyridine H), $8.95(\mathrm{~s}, 1 \mathrm{H}, \mathrm{CH}), 12.22(\mathrm{~s}, 1 \mathrm{H}, \mathrm{NH})(\mathrm{Fig} . \mathrm{S} 18) .{ }^{13} \mathrm{C}$ NMR : $121.9,128.4,130.6,133.1,140.8,148.1,150.8,162.2$ (Fig. S19). MS: m/z $373\left((\mathrm{M}+\mathrm{H})^{+}\right.$.

\section{In-vitro Evaluation of Synthesized Derivatives for Anti-mycobacterial Susceptibility}

All the mycobacterial works were done under the required biosafety level facility in the mycobacteriology biosafety laboratory with a required condition. Drug susceptibility testing of series of synthesized INH compounds against $M$. tuberculosis $\mathrm{H} 37 \mathrm{Rv}$ strain was analyzed using BACTEC MGIT ${ }^{\mathrm{TM}} 960$ instruments (Becton Dickinson Diagnostic Systems, Sparks, MD, USA). Prescribed manufacturer's guidelines for frontline drugs was strictly followed for testing drug susceptibility. MGIT ${ }^{\mathrm{TM}}$ tube contains $7 \mathrm{~mL}$ of Middlebrook 7H9 broth and an oxygen-quenching fluorescent sensor system is fixed in silicone at the bottom to represent the oxygen concentration based on organic activity. To explain the above-said experiment, on confirmation of positive growth by BACTEC instrument, Antimicrobial susceptibility Test (AST) was carried out on the $5^{\text {th }}$ day. To perform the AST, $0.8 \mathrm{ml}$ of oleic acid-albumin-dextrose (OADC) supplement was added to each MGITTM tube. $0.5 \mathrm{~mL}$ of positive broth culture, dilution at the rate of 1:100 was added to the Growth control tube (GC) and a dilution of 1:5 was added to other tubes. $100 \mu$ of synthesized derivatives and standard INH were added to the individual test tubes as labeled except for the control tube. The tubes were placed in AST rack in proper order and incubated at $37^{\circ} \mathrm{C}$ until the instrument signaled a beep. To determine the susceptibility results, the fluorescence of the drug-containing tubes in comparison to the GC tube was analyzed by the instrument concentrations. ${ }^{50}$ Further Confirmation of the culture M. tuberculosis was done using antigen kit MPT64.

\section{Drug Stability Test for Synthesized Biologically Active INH Derivatives}

Synthesized active derivatives were analyzed by Reverse Phase High-Performance Liquid Chromatography (RP-HPLC) system (Shimadzu, Japan) with an autosampler and UV detector. Phenomenex C18 Luna (150 $\times 4.6 \mathrm{~mm} ; 5 \mu \mathrm{m})$ column was used. Methanol and Water were used as mobile phases. HPLC grade chemicals and reagents were used for the entire analysis. Synthesized products were eluted by changing the aqueous: organic modifier ratio of solvents in the gradient form, absorbance was checked by UV spectrophotometer initially and two wavelengths 268 and $310 \mathrm{~nm}$ were set for HPLC method keeping all other instrumental conditions constant. The stability of the synthesized products was studied at the physiological condition of $\mathrm{pH} 7.4$ and acidic conditions at $\mathrm{pH} 4.5$. The procedure is reported in ${ }^{24}$. In brief, the appropriate dilutions of synthesized derivatives were made as per the solubility of the compounds (5a, $\mathbf{5 c}, \mathbf{5 d}, \mathbf{5 k}, \mathbf{5 l}, \mathbf{5 m})$ and analyzed by developed RP-HPLC method. The samples were kept on Rotary shaker and incubated at $37^{\circ} \mathrm{C}$. After certain time intervals, the samples were taken from the incubator and injected to HPLC to find the concentration of the pure drug. This RP-HPLC methods are simple, authentic and separate all the degradation products from active derivatives even at their lowest concentration. ${ }^{51}$

\section{Cytotoxic Effect Study on Macrophage Cells}

RAW 264.7 macrophage cell lines were obtained from National Centre for Cell Science (NCCS), Pune, India and Cells were maintained in DMEM medium with $10 \%$ fetal bovine serum (FBS) and antimicrobial mixture $1 \%$. Cells were cultured and retained at $37{ }^{\circ} \mathrm{C}$ and $5 \% \mathrm{CO}_{2} .5000$ cells for each well were seeded in plates (96 well) and left to adhere (Incubation). The treatment added were INH $0.1 \mu \mathrm{g} / \mathrm{ml}$ (MIC), synthesized active derivatives (Equivalent to MIC of INH $0.1 \mu \mathrm{g} / \mathrm{ml}$ ). MTT assay was carried out after 24 and 48 hours of treatment, using the optimised protocol. Briefly, the supernatant was taken out from each well and MTT $(5 \mathrm{mg} / \mathrm{mL})$ was put into the wells and left for $4 \mathrm{~h}$ incubation. MTT was taken out and DMSO $100 \mu \mathrm{L}$ was placed in each well after incubation. The UV absorbance at $570 \mathrm{~nm}$, readings were taken using a multi-plate reader. Each sample was recorded for cell viability. 
RASĀYAN J. Chem.

Vol. 13 | No. 3 |1853-1870| July - September | 2020

\section{Molecular Docking Analysis}

\section{RESULTS AND DISCUSSION}

The docking model was authenticated using the known structure of InhA bound to its ligand INH. The root mean square (RMS) deviation was $0.370 \AA$ between the predicted and actual pose, that was fine within the standard limit of $2.0 \AA .^{52}$ The docking models for INH derivatives revealed their interaction with target InhA, which is vital to catalyze the $\beta$-nicotinamide adenine dinucleotide (NADH)-specific reduction of 2trans-enoyl-acyl carrier protein. In that respect, to hinder the function of InhA, the docking models indicated by the suitable evidence about the energy, affinity and positioning of ligand-enzyme interactions. The docking method was authenticated using the reported structure of InhA bound to its ligand INH. The root mean square (RMS) deviation was $0.370 \AA$ between the predicted and actual pose, which was fine within the standard limit of $2.0 \AA^{52}$.

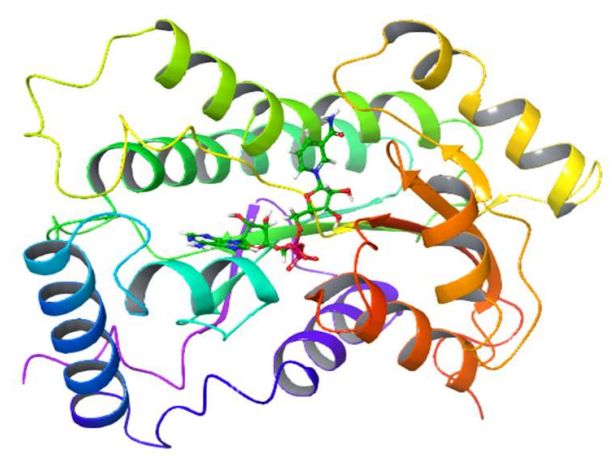

Fig.-1: Crystal Structure of the INH target of M. tuberculosis InhA protein

Table-1: Interaction Residues and Docking Scores for the Binding of INH Derivatives with InhA.

\begin{tabular}{c|c|c|c|c}
\hline Compound & $\begin{array}{c}\text { Docking } \\
\text { Score } \\
(\mathrm{kcal} / \mathrm{mol})\end{array}$ & $\begin{array}{c}\pi-\pi \text { Stacking } \\
\text { or } \pi \text { - Cation Interaction }\end{array}$ & $\begin{array}{c}\text { H-Bond forming } \\
\text { Residues }\end{array}$ & $\begin{array}{c}\text { Hydrophobic Interaction } \\
\text { Residues }\end{array}$ \\
\hline $5 \mathrm{a}$ & -5.3 & Phy41 & Val65 & Ile16, Ile21, Ile95, Ala198 \\
\hline $5 \mathrm{c}$ & -6.4 & Lys165 & Ile16, Ile21, Phe41, Ile95 \\
\hline $5 \mathrm{~d}$ & -6.5 & Phy41 & Gly14, Gly96 \& Thr196 & Ile16, Ile21, Val65, Ile95 \\
\hline $5 \mathrm{k}$ & -7.8 & Phy41 & Val65, Gly96 \& Thr196 & Ile16, Ile21, Ile95, Met199 \\
\hline 51 & -7.4 & Phy41 & Val65, Ser94 & Ile16, Ile21, Ile95 \\
\hline $5 \mathrm{~m}$ & -7.9 & Phy41 \& Phe97 & Gly14, Gly96 \& Thr196 & Ile16, Ile21, Ile95, Ala198 \\
\hline $\mathrm{INH}$ & -5.0 & Phy41 & Val65 & Phe41, Ile95 \\
\hline
\end{tabular}

The X-ray crystallography determines the docking process precision. Glide Score (GScore) virtually features this experimental binding mode ${ }^{53}$ The pIC 50 of many inhibitors retain in contact with the Glide score as well as MM-GBSA values as shown in Table-1. The docking model demonstrated the stacking interaction of INH derivatives through their hydrophobic and aromatic groups such as Indole/pyridine and imidazole rings in the active site formed by amino acids, Gly 14 to Thr196. INH analogs (5a-m) displayed a network of hydrogen bonding with Val65, Ser94, Gly96, Thr196 residues belongs to the catalytic-domain of InhA protein. As displayed, the docked pose of this most active compound $\mathbf{5 l} N^{\prime}$-[(2-butyl-4-chloro- $1 \mathrm{H}-$ imidazol-5-yl)methylidene]pyridine-4- carbohydrazide) among the active site of 1ENY (Fig.-2), Imidazole/pyridine group position one $\mathrm{NH}$ formed a hydrogen-bonding system along with the backbone 
RASĀYAN J. Chem.

Vol. 13 | No. 3 |1853-1870| July - September | 2020

amine Val 65 group $(2.08 \AA)$ and even an additional hydrogen bonding was noticed between $1 \mathrm{NH}$ moiety of $1 \mathrm{H}$-imidazole with backbone group amide of Ser94 (2.99 $\AA$ ). The interaction of compounds $\mathbf{5 a}, \mathbf{5} \mathbf{k}$ and $\mathbf{5 l}$ with the catalytic domain of InhA protein found similar to the interaction of INH 3 (Fig.-2a-g).

The RMS deviation among the conformation predicted and the detected (X-ray crystallographic) conformation of INH (PDB 1ENY) of $0.370 \AA$ (Fig.-3), this value pronounces the stability of InhA/inhibitor complexes.
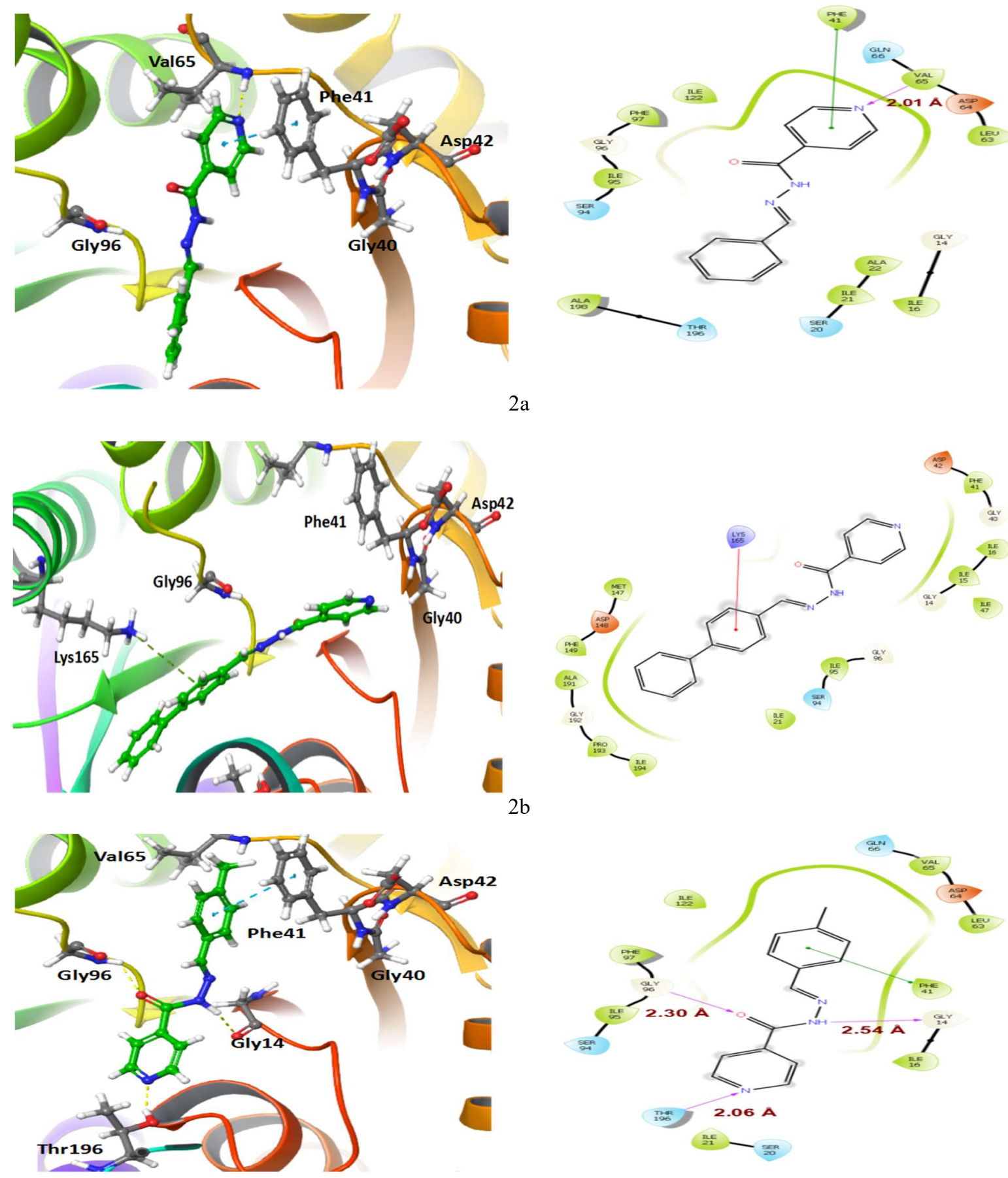
RASĀYAN J. Chem.

Vol. 13 | No. 3 |1853-1870| July - September | 2020
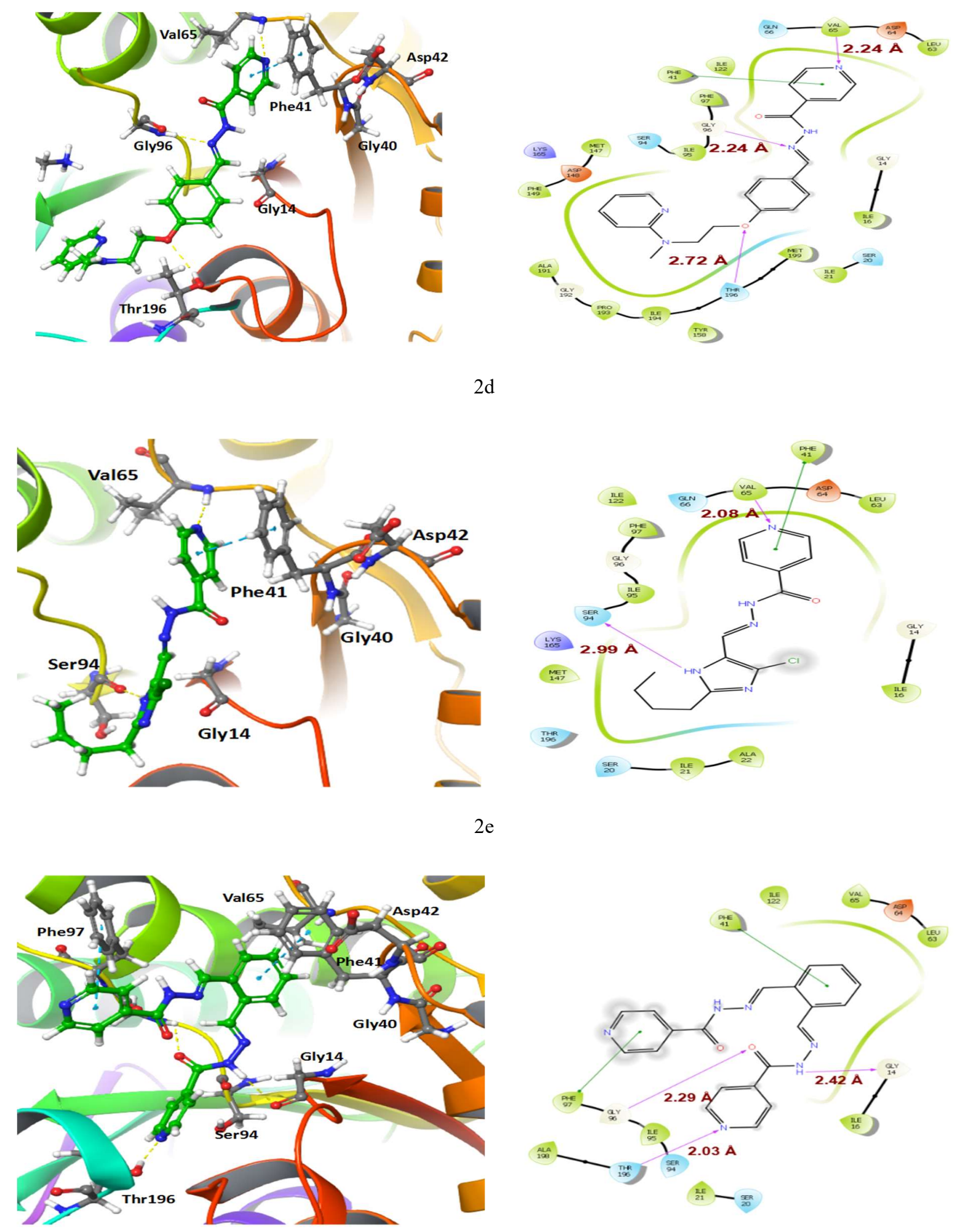
RASĀYAN J. Chem.

Vol. 13 | No. 3 |1853-1870| July - September | 2020

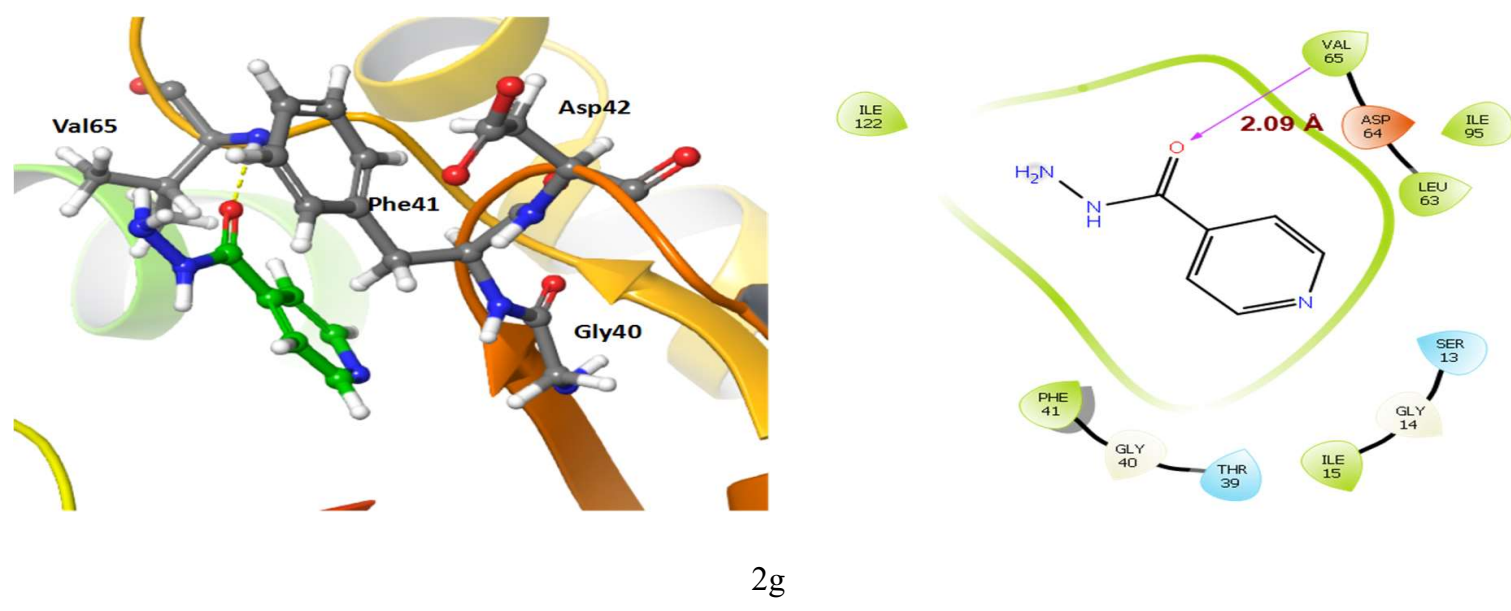

Fig.-2: Binding Mode of Compounds in the Catalytic Pocket of InhA (1ENY). 2a: Interaction of Compound 5a in 3D Space and 2D Protein Ligand Interaction, 2b: Interaction of Compound 5c in 3D Space and 2D Protein Ligand Interaction, 2c: Interaction of Compound 5d in 3D Space and 2D Protein Ligand Interaction, 2d: Interaction of Compound 5k in 3D Space and 2D Protein Ligand Interaction, 2e: Interaction of Compound 51 in 3D Space and 2D Protein Ligand Interaction, 2f: Interaction of Compound 5m in 3D Space and 2D Protein Ligand Interaction, 2g: Interaction of Compound INH (3) in 3D Space and 2D Protein Ligand Interaction.

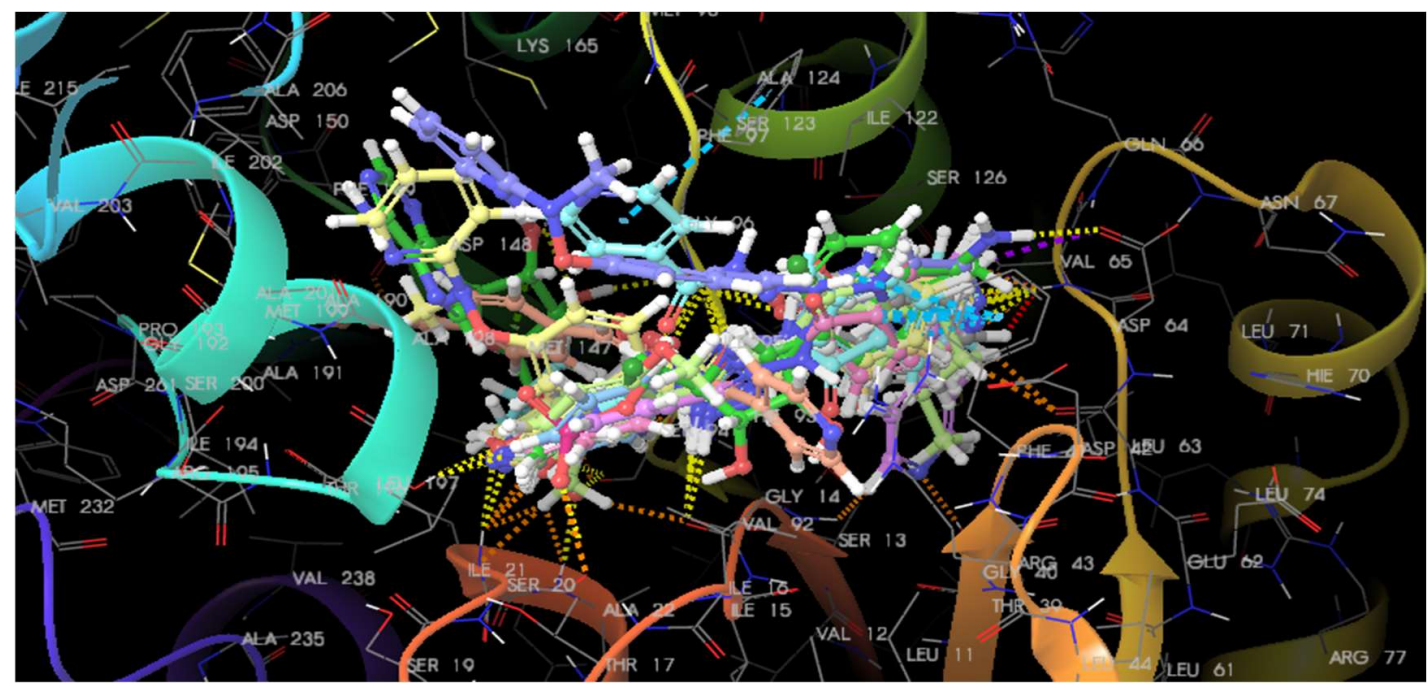

Fig.-3: Binding Mode of All Compounds in the Catalytic Pocket of InhA (1ENY) and Active Site Occupancy.

\section{Molecular Dynamics Study}

Simulation studies were done by the docked conformation of 51/1ENY complex which interacts with Ser94 (susceptible for substitution with alanine), to study the atomic information of molecular interactions. RMS deviation from the initial structure was done to analyze the system's dynamic stability. The RMS deviation values of $1 \mathrm{ENY}$ backbone were $1.65,1.62$ and $1.84 \AA$, demonstrating that, the stability of the protein did not vary intensely during its process of changing its structure (from crystal to protein) in water. Out of total 10,1000 structures computed were stored in the trajectory Last simulation period was for a total of $10 \mathrm{~ns}$. Deviations amongst the original and the structure obtained by simulation were plotted as shown in Fig.-4. During the simulation, protein backbone RMS deviation displayed a huge deviation for the initial $100 \mathrm{ps}$ due to the initial structural stabilization of the protein; later displayed stable state dynamics. Backbone structural deviations seen for the latter phase of 2 to $10 \mathrm{~ns}$ ranged from $1.8 \AA$ to $2.4 \AA$ related to that of the original structure. The total RMS deviation of the receptor from $2 \mathrm{~ns}$ to the end was within a range of $0.6 \AA$ which is an acceptable range of deviation. Fig.-4 shows the RMS deviation of the ligand 51 which has 
RASĀYAN J. Chem.

Vol. 13 | No. 3 |1853-1870| July - September | 2020

reduced to $1.2 \AA$ after $2 \mathrm{~ns}$ simulation then stabilized. This strongly validates that in the protein binding site the ligand is well stabilized.

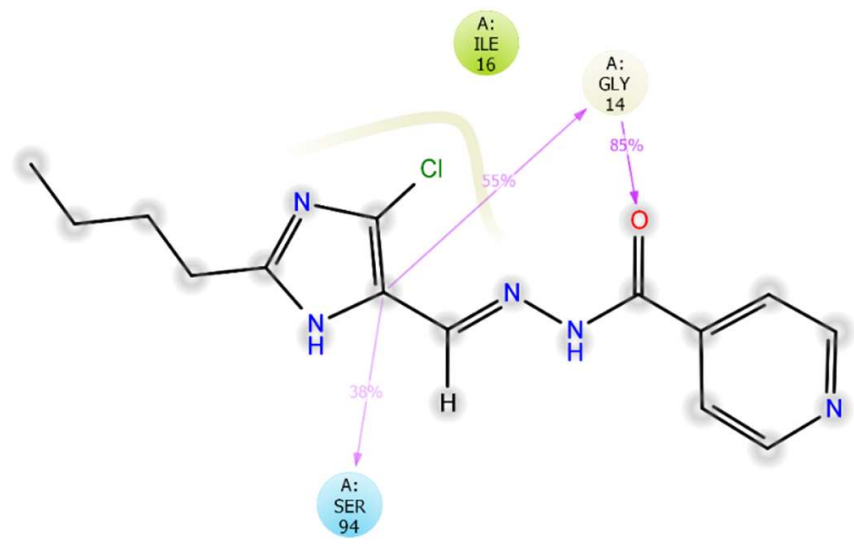

Fig.-4: Represents the RMS Deviation ( $\AA$ ) of the Simulated Positions of 1ENY Backbone Atoms From those in the Initial Structure

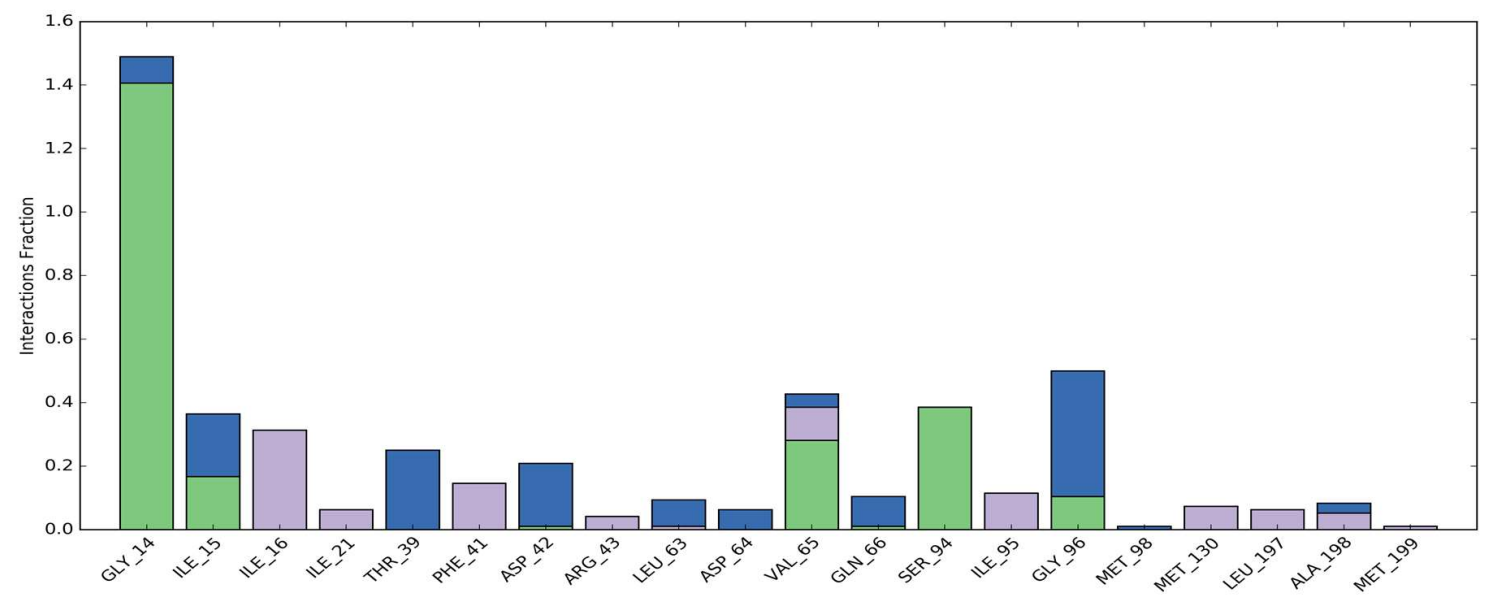

Fig.-5: Interaction between InhA and 51 evolved during MD simulation.

Figure-5 displays results of the entire interactions detected throughout the docking simulation for compounds 51. All the ligand-protein interactions seen during the analysis remained all through the docking simulation.

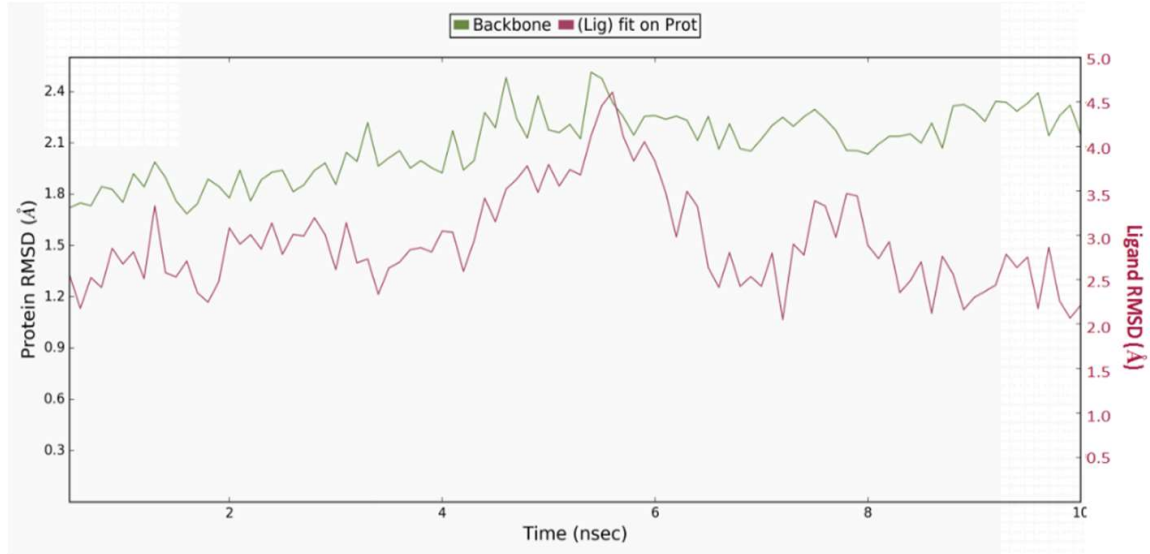

Fig.-6: Schematic Illustration of Ligand Interaction with the Amino Acid Residues of Protein During Docking Simulation. 
RASĀYAN J. Chem.

Vol. 13 | No. 3 |1853-1870| July - September | 2020

The exact interactions protein made with the ligand over the sequence of the trajectory total number is shown in Fig.-5. The structure developed from the simulation, clearly indicates that H-bond with Gly 14 and Ser94, the $\pi-\pi$ interaction with Phy 41 are donating strongly for the binding affinity. More than $25 \%$ of the simulation time interactions are shown in Fig.-6.

\section{Chemistry}

The isonicotinic acid was esterified using ethanol and a catalytic amount of sulphuric acid. INH is obtained by treating ethyl isonicotinate with hydrazine hydrate in ethyl alcohol medium. INH was further treated with substituted aromatic/ heteroaromatic aldehydes 4 (a-l) to achieve a good yield of corresponding hydrazones 5 (a-l) (Scheme-1). The purity of the synthesized hydrazones 5 (a-1) are determined by HPLC and are characterized using FTIR, ${ }^{1} \mathrm{HNMR},{ }^{13} \mathrm{C}$ NMR and Mass spectral techniques. The characteristic peaks observed in the infrared spectrum are, $\mathrm{C}=\mathrm{N}$ and $\mathrm{C}=\mathrm{O}$ stretching in the region $1488-1596 \mathrm{~cm}^{-1}$ and $1660-1680 \mathrm{~cm}^{-1}$ respectively. ${ }^{1} \mathrm{H}$ NMR indicates the characteristic signals at $\delta 8.4-8.6,6.9-8.5$ and $12-12.5$ due to $-\mathrm{CH}=\mathrm{N}$, aromatic protons and $-\mathrm{N} H$ protons respectively which indicates the formation of the product. In ${ }^{13} \mathrm{C}$ NMR spectrum, imine carbon was observed at around $\delta 149$ and carbonyl carbon was detected at $\delta 160-162$. All other peaks were seen in the predictable region. The formation of the product was further established by Mass spectral analysis.<smiles>CCOC(=O)c1ccccc1OCC</smiles>

1<smiles>CCOC(=O)c1ccncc1</smiles>

2

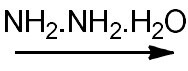<smiles>NNC(=O)c1ccncc1</smiles>

3<smiles>[R]C=NNC(=O)c1ccncc1</smiles>

$5(a-m)$

Where

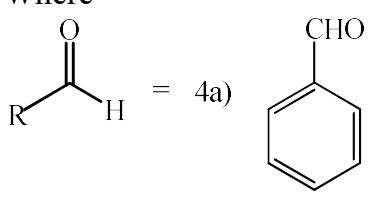

4b)<smiles>O=Cc1cccc([N+](=O)[O-])c1</smiles>

4g)<smiles>O=Cc1ccccc1O</smiles>

4h)

)<smiles>O=Cc1ccc(-c2ccccc2)cc1</smiles>
4d)<smiles>Cc1ccc(C=O)cc1</smiles>

4e)<smiles>O=Cc1ccc(Cl)cc1</smiles><smiles>COc1ccc(I)cc1C=O</smiles>

4f)<smiles>O=Cc1ccc(F)cc1</smiles>

4i)<smiles>O=Cc1ccc(O)c(O)c1</smiles><smiles>O=Cc1c[nH]c2ccccc12</smiles>

$4 \mathrm{k})$<smiles>CN(CCOc1ccc(C=O)cc1)c1ccccn1</smiles><smiles>CCCCc1nc(Cl)c(C=O)[nH]1</smiles>

$4 \mathrm{~m})$<smiles>O=Cc1ccccc1C=O</smiles>

Scheme-1: Synthesis of Isoniazid Hydrazones 
RASĀYAN J. Chem.

Vol. 13 | No. 3 |1853-1870| July - September | 2020

\section{In vitro Antimycobacterial Susceptibility Evaluation}

The biological assessment of all the synthesized INH derivatives was determined against $M$. tuberculosis H37Rv strain in Middlebrook 7H9 medium using a MGITTM 960 instrument. $0.1 \mu \mathrm{g} / \mathrm{mL}$ of INH is the Minimum Inhibitory Concentration (MIC), which is essential to arrest the total growth of H37Rv strain. Therefore, MIC is taken as the standard and equivalent molar concentration of synthesized INH derivatives were evaluated for AST, as part of standard procedure. Results are shown in Table 2, in which INH derivatives $\mathbf{5 a}, \mathbf{5 c}, \mathbf{5 d}, \mathbf{5 k}, \mathbf{5 l}$ and $\mathbf{5 m}$ showed sensitivity, which means compounds are efficient in inhibiting the growth of M. tuberculosis H37Rv strain completely. Remaining tubes $\mathbf{5 b}, \mathbf{5 e}, \mathbf{5 f}, \mathbf{5 g}, \mathbf{5 h}, \mathbf{5 i}, \mathbf{5 j}$ showed resistance to AST, it means the compounds were not able to prevent the growth of tested strain completely by the given MIC concentration. The increase of fluorescence represents the consumption of oxygen by the actively respiring organism. The instrument automatically reads and records the drug susceptibility results. Instrument defines the results of the test samples in comparison with the fluorescence of the control tube (Fig.-7) which showed a growth unit. Benzohydrazone 5a, biphenyl hydrazone 5c and 4-methyl benzohydrazone $\mathbf{5 d}$ compounds inhibited the growth of M. tuberculosis. Similarly, the compounds $\mathbf{5 k}$ and 5l containing alkyl substitution on aryl and heteroaryl position showed positive to AST by completely inhibiting the growth of $\mathrm{H} 37 \mathrm{Rv}$ strain of M. Tuberculosis. INH derivatives having nitro, chloro, hydroxy and methoxy substituents of benzohydrazones showed resistance. The structural activity correlates mycobacterial growth inhibition was seen in compounds with the presence of aryl heteroaryl group or alkyl substitution on aryl/heteroaryl groups. The presence of nitro, chloro, hydroxy and methoxy substituents on aryl/heteroaryl moieties subsides the antimycobacterial activity. Results suggest that the compounds with hydrophobic groups exhibited potent anti-tubercular activity being sensitive towards $M$. tuberculosis by completely inhibiting the growth. Results were confirmed in triplicates. Whereas reducing the drug concentration of these compounds failed to prevent the growth of M. tuberculosis completely. Similarly, the hydrophilicity of derivatives exhibits reduced activity showing resistance.

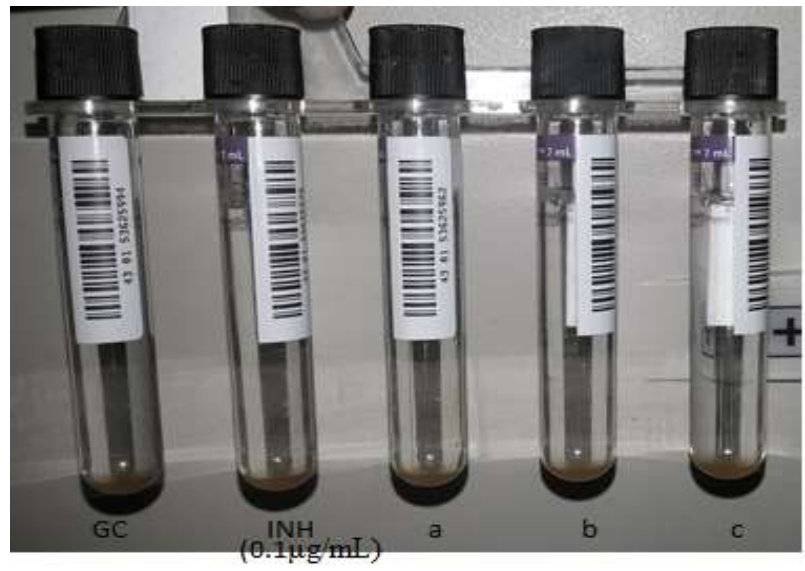

Fig.-7: AST against M. tuberculosis H37Rv, GC has Positive Growth Control, a Second Tube with INH at its MIC $0.1 \mu \mathrm{g} / \mathrm{mL}$, a-c Test Samples in Triplicates

\section{Results of Drug Stability Tests for Synthesized Biologically Active derivatives}

The stability graphs at different $\mathrm{pH}$ environment were plotted as shown in Fig.-8 and Fig.-9. Results indicated that when standard INH and its synthesized biologically active derivatives were subjected to acidic and basic $\mathrm{pH}$ solutions at $37^{\circ} \mathrm{C}$, synthesized derivatives remained more stable in comparison with standard INH. 5a and $\mathbf{5 c}$ compounds exhibited good stability around $85 \%$ up to $48 \mathrm{~h}$ in normal blood pH conditions whereas $\mathbf{5 d}, \mathbf{5 k}, \mathbf{5 m}$ compounds exhibited 60 to $70 \%$ stability at the same condition $7.4 \mathrm{pH}$ (Fig.8). Whereas stability of INH declined to $60 \%$ and below at $48^{\text {th }}$ hour and almost disappeared thereafter at this basic $\mathrm{pH}$ condition.

In the case of acidic pH, only $5 \%$ of fall instability was observed with standard INH (Fig.-9). Whereas the degradation rate of the synthesized derivatives varied from $10 \%$ to $50 \%$ in 48 hours at the $4.5 \mathrm{pH}$ condition. 
RASĀYAN J. Chem.

Vol. 13 | No. 3 |1853-1870| July - September | 2020

Developed RP-HPLC analytical method proved very effective in depicting the minute separations of drugs and products. The variations seen in drug stability at various $\mathrm{pH}$ conditions and other constants shows that a higher concentration of drugs needs to be administered to reach the site of infection for therapeutic action. This leads to severe side effects. Hence, maintaining high stability and increasing the bioavailability of drugs by decreasing the degradation rate place a crucial factor in TB treatment.

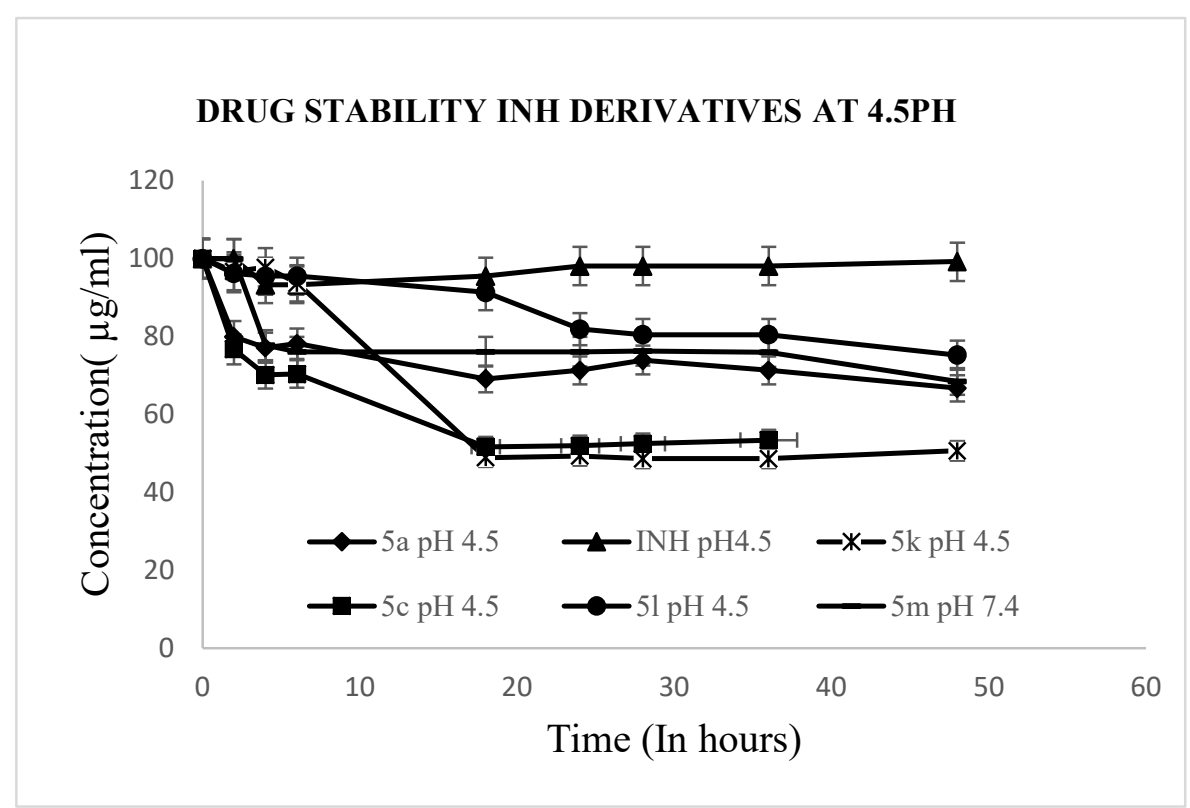

Fig.-8: INH Derivatives Drug Stability Test at 7.4pH Comparison with INH

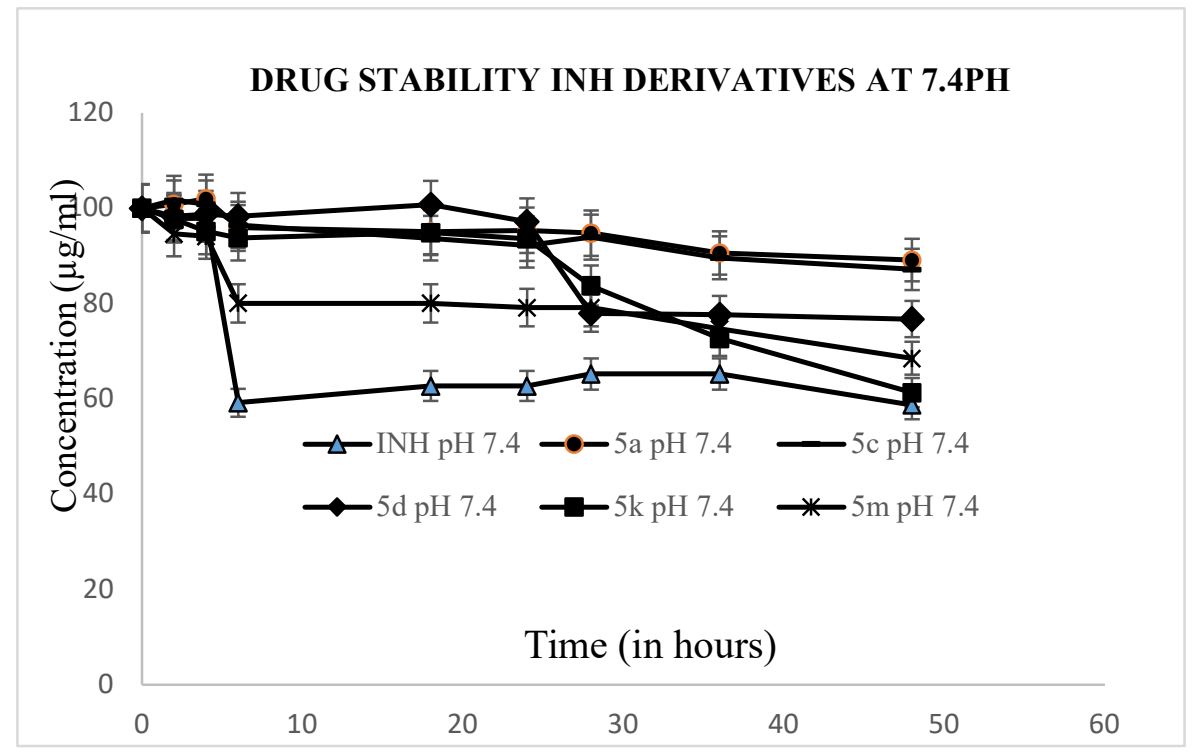

Fig.-9: INH Derivatives Drug Stability Test at $4.5 \mathrm{pH}$ Comparison with INH

\section{Cytotoxic Effect Study on Macrophage Cells}

The toxic effects of drug concentrations on RAW 264.7 macrophage cells through MTT assays were analyzed. Cells were subjected to MIC of INH and its equivalent of the molar concentration of synthesized derivatives for 24 hours and 48 hours, the cell viability remained unchanged as shown in Fig.-10 and Fig.11. These results suggest that synthesized drugs have no toxic effect on the RAW 264.7 macrophage cells. 
RASĀYAN J. Chem.

Vol. 13 | No. 3 |1853-1870| July - September | 2020

Table-2: Anti-mycobacterial Susceptibility Test Results

\begin{tabular}{c|c|c|c|c}
\hline Tube Position & $\begin{array}{c}\text { Growth } \\
\text { Unit }\end{array}$ & Status & $\begin{array}{c}\text { Drug Conc. } \\
(\mu \mathrm{g} / \mathrm{ml})\end{array}$ & Samples \\
\hline B/R06 & 400 & $\mathrm{C}$ & - & Growth Culture \\
\hline B/R07 & 0 & $\mathrm{~S}$ & 0.1 & $\mathrm{INH}$ \\
\hline $\mathrm{B} / \mathrm{R} 12$ & 0 & $\mathrm{~S}$ & 0.1 & $5 \mathrm{a}$ \\
\hline $\mathrm{B} / \mathrm{S} 13$ & 400 & $\mathrm{R}$ & 0.1 & $5 \mathrm{~b}$ \\
\hline $\mathrm{B} / \mathrm{R} 13$ & 0 & $\mathrm{~S}$ & 0.1 & $5 \mathrm{c}$ \\
\hline $\mathrm{B} / \mathrm{R} 18$ & 0 & $\mathrm{~S}$ & 0.1 & $5 \mathrm{~d}$ \\
\hline $\mathrm{B} / \mathrm{S} 13$ & 400 & $\mathrm{R}$ & 0.1 & $5 \mathrm{e}$ \\
\hline $\mathrm{B} / \mathrm{B} 08$ & 400 & $\mathrm{R}$ & 0.1 & $5 \mathrm{f}$ \\
\hline $\mathrm{B} / \mathrm{B} 13$ & 400 & $\mathrm{R}$ & 0.1 & $5 \mathrm{~g}$ \\
\hline $\mathrm{B} / \mathrm{B} 18$ & 400 & $\mathrm{R}$ & 0.1 & $5 \mathrm{~h}$ \\
\hline $\mathrm{B} / \mathrm{C} 04$ & 400 & $\mathrm{R}$ & 0.1 & $5 \mathrm{i}$ \\
\hline B/C08 & 400 & $\mathrm{R}$ & 0.1 & $5 \mathrm{j}$ \\
\hline B/R08 & 0 & $\mathrm{~S}$ & 0.1 & $5 \mathrm{k}$ \\
\hline B/S03 & 0 & $\mathrm{~S}$ & 0.1 & $5 \mathrm{l}$ \\
B/B06 & 0 & $\mathrm{~S}$ & 0.1 & $5 \mathrm{~m}$ \\
\hline
\end{tabular}

C- Control, S- Sensitive, R-Resistant, INH-Isoniazid

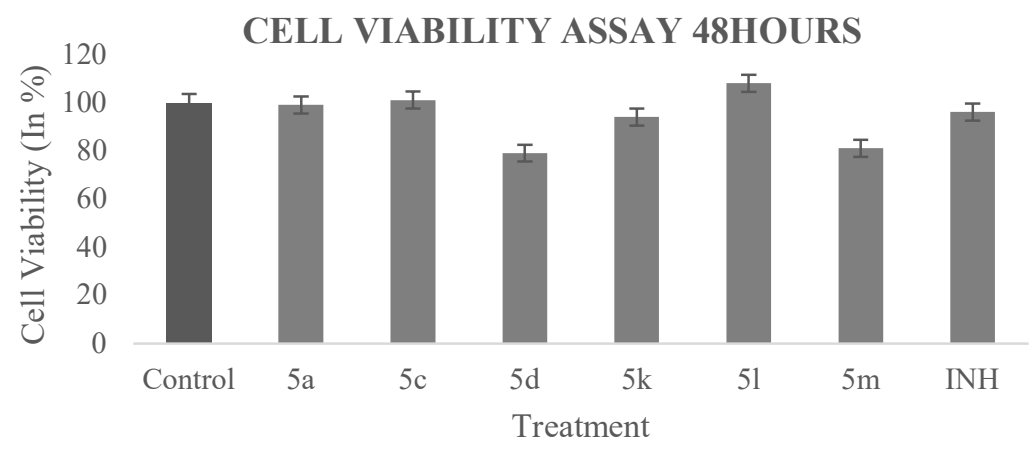

Fig.-10: Cytotoxic Effect after 24 Hours Treatment on Macrophage Cells RAW 264.7

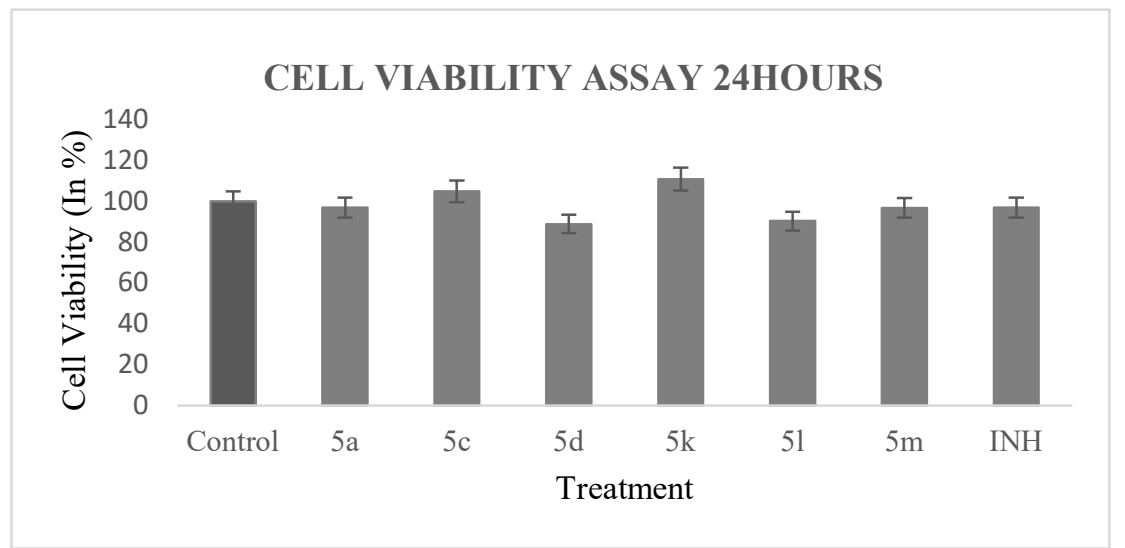

Fig.-11: Cytotoxic Effect after 48 Hours Treatment on Macrophage Cells RAW 264.7

\section{CONCLUSION}

Among the designed series of INH derivatives, compounds reported herein, Benzohydrazone 5a, biphenyl hydrazone $\mathbf{5 c}$ and N'-[(4-methylphenyl)methylidene]pyridine-4-carbohydrazide $\quad \mathbf{5 d}), \quad$ N'-[(4-\{2- 
RASĀYAN J. Chem.

Vol. 13 | No. 3 |1853-1870| July - September | 2020

[methyl(pyridin-2-yl)amino] ethoxy\}phenyl)methylidene]pyridine-4-carbohydrazide (5k), N'-[(2-butyl-5chloro-1H-imidazol-4-yl) methylidene]pyridine-4-carbohydrazide (5l) containing alkyl substitution on aryl and heteroaryl position, similarly N'-[phenyl 1,2-dimethylidene]pyridine-4-carbohydrazide (5m) which are modified with hydrophobic groups expressed greater stability without disturbing their therapeutic efficacy. Compounds mentioned above $\mathbf{5 a}, \mathbf{5 c}, \mathbf{5 d}, \mathbf{5 k}, \mathbf{5 l}, \mathbf{5 m}$ indicated sensitivity by inhibiting the growth of H37Rv strain at the molar equivalent of Minimum Inhibitory Concentration of INH. Cytotoxicity results revealed that synthesized active INH derivatives do not pose any toxic effect on RAW 264.7 macrophage cell line. The compounds N'-[(3-nitrophenyl)methylidene]pyridine-4-carbohydrazide(5b), N'-[(4chlorophenyl)methylidene]pyridine-4-carbohydrazide(5e), N'-[(4-fluorophenyl)methylidene]pyridine-4carbohydrazide(5f), $\quad \mathrm{N}^{\prime}-[(2$-hydroxyphenyl)methylidene]pyridine-4-carbohydrazide(5g), $\quad$ N'-[(2,5dimethoxyphenyl)methylidene]pyridine-4-carbohydrazide(5h), N'-[(3,4-dihydroxyphenyl) methylidene]

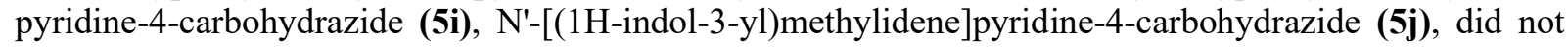
show inhibition of growth of H37Rv strain at molar equivalent of Minimum Inhibitory Concentration of INH.

\section{ACKNOWLEDGMENT}

Authors acknowledge to the Central Instrumentation Facility, MAHE, Manipal and Atrimed Pharmaceuticals Pvt. Ltd. Bangalore. Vision Group of Science and Technology (VGST), Karnataka for funding granted to Dr. Bharath Raja Guru.

1. J.B. Silva Jr, Jornal Brasileiro de Pneumologia, 30, S57(2004), DOI:10.1590/S180637132004000700003

2. H.M. Blumberg, W.J. Burman, R.E. Chaisson, C.L. Daley, S.C. Etkind, L.N. Friedman, P. Fujiwara, M. Grzemska, P.C. Hopewell, M.D. Iseman, R.M. Jasmer, V. Koppaka, R.I. Menzies, R.J. O'Brien, R.R. Reves, L.B. Reichman, P.M. Simone, J.R Starke and A.A. Vernon, American Journal of Respiratory and Critical Care Medicine, 167, 603(2003), DOI:10.1164/rccm.167.4.603

3. R. Shegokar, A. Loaye and M. Khalil, Journal of Pharmacy and Pharmaceutical Sciences, 14, 100 (2011), DOI: $10.18433 / \mathrm{j} 3 \mathrm{~m} 59 \mathrm{p}$

4. H.M. Faidalla, K.A. Khan and M.S.I. Makki, Journal of the Chinese Chemical Society, 58, 191(2011), DOI: $10.1002 /$ jecs.201190076

5. S.S. Bishta, A. Arya, K.S. Sudhir, C. Vinita and P.T. Rama, International Journal of Drug Design and Discovery, 1, 11(2010).

6. A. Omar and M.A. Ahmed, World Applied Sciences Journal, 5, 94(2008).

7. R.P. Tripathi, N. Tewari, N. Dwivedi and V.K. Tiwari, Medicinal Research Reviews, 25, 93(2005), DOI: $10.1002 /$ med.20017

8. J. Bernstein, W.A. Lott, B.A. Steinberg and H.L. Yale, American Review of Respiratory Disease, 65, 357(1952), DOI: 10.1164/art.1952.65.4.357

9. C. Vilchèze and W.R. Jacobs Jr., Annual Review of Microbiology, 61, 35(2007), DOI: 10.1146/annurev.micro.61.111606.122346

10. F. Bardou, C. Raynaud, C. Ramos and M.A. Lanéelle, Microbiology, 144, 2539(1998), DOI: 10.1099/00221287-144-9-2539

11. D.A. Mitchison and J.B. Selkon, American Review of Tuberculosis, 74, 109(1956), DOI: https://www.atsjournals.org/doi/abs/10.1164/artpd.1956.74.2-2.109

12. F.G. Winder and P.B. Collins, Journal of General Microbiology, 63, 41(1970), DOI: $10.1099 / 00221287-63-1-41$

13. G.S. Timmins and V. Deretic, Molecular Microbiology, 62, 1220(2006), DOI:10.1111/j.13652958.2006.05467.x

14. K. Johnsson and P.G. Schultz, Journal of the American Chemical Society, 16, 7425(1994), DOI: 10.1021/ja00095a063

15. B.F. Lei, C.J. Wei and S.C. Tu, Journal of Biological Chemistry, 275, 2520(2000), DOI: $10.1074 / \mathrm{jbc} .275 .4 .2520$

16. A.N. Unissa, S. Hassan and N. Selvakumar, International Journal of Pharma and Bio Sciences, 3, $314(2012)$

STABLE ISONIAZID DERIVATIVES 
RASĀYAN J. Chem.

Vol. 13 | No. 3 |1853-1870| July - September | 2020

17. S.K. Sridhar, S.N. Pandeya, J.P. Stables and A. Ramesh, European Journal of Pharmaceutical Sciences, 16, 129(2002), DOI:10.1016/s0928-0987(02)00077-5

18. E.E. Oruc, B. Kocyigit-Kaymakcioglu, B. Oral, H.Z. Altunbas-Toklu, L. Kabasakal and S. Rollas, Archiv der Pharmazie - Chemistry in Life Sciences, 339, 267(2006), DOI:10.1002/ ardp.200500202

19. N. Sinha, S. Jain, A. Tilekar, R.S. Upadhayaya, N. Kishore and G.H. Jana, Bioorganic \& Medicinal Chemistry Letters, 15, 1573(2005), DOI:10.1016/j.bmcl.2005.01.073

20. S. Kakimoto and K. Yamamoto, Chemical and Pharmaceutical Bulletin, 4, (1956), DOI: $10.1248 / \mathrm{cpb} 1953.4 .4$

21. S.E. Helaly, A. Pesic, H.P. Fiedler and R.D. Sussmuth, Organic Letters, 13, 1052(2011), DOI: $10.1021 / 011031014$

22. S.J. Gilani, K. Nagarajan, D.P. Maurya, D. Katiyar, R. Goel and S.A. Khan, Medicinal Chemistry, 4, 428(2014), DOI: 10.4172/2161-0444.1000174

23. Y.Q. Hu, S. Zhang, F. Zhao, C. Gao, L.S. Feng, Lv. Zao-Sheng, Z. Xu and X. Wu, European Journal of Medicinal Chemistry, 133, 255(2017), DOI:10.1016/j.ejmech.2017.04.002

24. S.S. Hakkimane, V. P. Shenoy, S. L. Gaonkar, I. Bairy and B.R. Guru, International Journal of Nanomedicine, 13, 4303(2018), DOI:10.2147/IJN.S163925

25. S.L. Gaonkar and K.M.L. Rai, B. Prabhuswamy, European Journal of Medicinal Chemistry, 41, 841 (2006), DOI:10.1016/j.ejmech.2006.03.002

26. K.H. Sunitha, S. L. Gaonkar, S.M. Sebastin, M.S. Kumar, Basappa, B.S. Priya, K. Kemparaju, K.S.Rangappa, S. Nanjunda Swamy and K.S. Girish, Basic and Clinical Pharmacology and Toxicology, 109, 292(2011), DOI:10.1111/j.1742-7843.2011.00725.x

27. E. Aparna, K. M. L. Rai, M. Sureshbabu, R. L. Jagadish, S. L. Gaonkar and K. Byrappa, Journal of Materials Science, 41, 1391 (2006), DOI:10.1007/s10853-006-7412-z

28. S. L. Gaonkar and K.M.L. Rai, Tetrahedron Letters, 46, 5969(2005), DOI: 10.1016/j.tetlet.2005.06.007

29. B. Namratha, D. Bilehal, V. ShyamKumar and S. L. Gaonkar, Research on Chemical Intermediates, 42, 1885(2016), DOI:10.1007/s11164-015-2123-1

30. A. Somoskovi, Tuberculosis., 95, 197(2015), DOI:10.1016/j.tube.2015.02.030

31. Revised National Tuberculosis Control Program, (2012), Accessed $1^{\text {st }}$ December 2016.

32. B.Lei and C.J. Wei and S.C. Tu., Journal of Biological Chemistry, 275, 2520(2000), DOI: $10.1074 / j b c .275 .4 .2520$

33. Y. Zhang and W.W. Yew, International Journal of Tuberculosis and Lung Disease, 19, 1276(2015), DOI: $10.5588 /$ ijtld.15.038

34. G.M. Sastry, M. Adzhigirey, T. Day, R. Annabhimoju and W. Sherman, Journal of Computer-Aided Molecular Design, 27, 221(2013), DOI: 10.1007/s10822-013-9644-8

35. M. P. Jacobson, D. L. Pincus, C. S. Rapp, T. J. F. Day, B. Honig, D. E. Shaw and R.A. Friesner, Proteins: Structure, Function, and Bioinformatics, 55, 35 (2004), DOI:10.1002/prot.10613

36. D. Shivakumar, J. Williams, Y. Wu, W. Damm, J. Shelley and W. Sherman, Journal of Chemical Theory and Computation, 6, 1509(2010), DOI:10.1021/ct900587b

37. R.A. Friesner, R.B. Murphy, M.P. Repasky, L.L. Frye, J.R. Greenwood, T.A. Halgren, P.C. Sanschagrin and D.T. Mainz, Journal of Medicinal Chemistry, 49, 6177(2006), DOI: $10.1021 / \mathrm{jm} 0512560$

38. J. Li, R. Abel, K. Zhu, Y. Cao, S. Zhao and R.A. Friesner, Proteins: Structure, Function, and Bioinformatics, 79, 2794(2011), DOI:10.1002/prot.23106

39. Z. Guo, U. Mohanty, J. Noehre, T.K. Sawyer, W. Sherman and G. Krilov, Chemical Biology \& Drug Design, 75, 348 (2010), DOI:10.1111/j.1747-0285.2010.00951.x

40. W.L. Jorgensen, J. Chandrasekhar, J.D. Madura, Impey, R.Wand M.L. Klein, Journal of Chemical Physics, 79, 926(1983), DOI:10.1063/1.445869

41. U. Essmann, L. Perera, M.L. Berkowit, T. Darden, H. Lee and L.G. Pedersen, Journal of Chemical Physics, 103, 8577(1995), DOI:10.1063/1.470117

42. G.J. Martyna, M.L. Klein, M. and Tuckerman, Journal of Chemical Physics, 97, 2635(1992), DOI: $10.1063 / 1.463940$ 
RASĀYAN $J$. Chem.

Vol. 13 | No. 3 |1853-1870| July - September | 2020

43. G.J. Martyna, D.J. Tobias and M.L. Klein, Journal of Chemical Physics, 101, 4177(1994), DOI: $10.1063 / 1.467468$

44. A.B. Thomas, P.N. Tupe, R.V. Badhe, R.K. Nanda, L.P. Kothapalli, O.D. Paradkar and P.A. Sharma and A.D. Deshpande, Green Chemistry Letters and Reviews, 2, 23(2009), DOI: $10.1080 / 17518250902922798$

45. A.B. Thomas, R.K. Nanda, L.P. Kothapalli, and A.D. Deshpande, Journal of the Korean Chemical Society, 55, 960(2011), DOI:10.5012/jkcs.2011.55.6.960

46. L.N. Suvarapu, Y-K. Seo and S-O. Baek, Chemical Science Transactions, 1, 171(2012), DOI: $10.7598 /$ cst2012.102

47. C. N. Nalini, Arivukkarasi and R. Devi, Rasayan Journal of Chemistry, 4, 868(2011).

48. A. Trzesowska-Kruszynska, Crystal Growth \& Design, 13, 3892(2013), DOI:10.1021/cg400529s

49. A. Chaouiki, H. Lgaz, R. Salghi, M. Chafiq, S. L. Gaonkar, K. S. Bhat, H. Oudda, I. H. Ali and I.-M. Chung, Research on Chemical Intermediates, 46, 2919(2020), DOI:10.1007/s11164-020-04119-6

50. S. Siddiqi, S. Rüsch-Gerdes, Foundation for Innovative New Diagnostics Ed, (2006)

51. S.S. Hakkimane and B.R. Guru, Asian Journal of Pharmaceutical and Clinical Research, 10, 330(2017), DOI:10.22159/ajpcr.2017.v10i5.17582

52. M. Kontoyianni, L. M. McClellan and G. N. Sokol, Journal of Medicinal Chemistry, 47, 558(2004), DOI: $10.1021 / \mathrm{jm} 0302997$

53. G. L. Card, L. Blasdel, B. P. England, C. Zhang, Y. Suzuki, S. Gillette, D. Fong, P. N. Ibrahim, D.R. Artis, G. Bollag, M.V. Milburn, S. H. Kim, J. Schlessinger and K.Y. Zhang, Nature Biotechnology, 23, 201(2005), DOI:10.1038/nbt1059

[RJC-5667/2020] 